\title{
Altered DNA Methylation in the Developing Brains of Rats Genetically Prone to High versus Low Anxiety
}

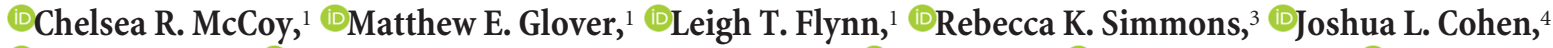

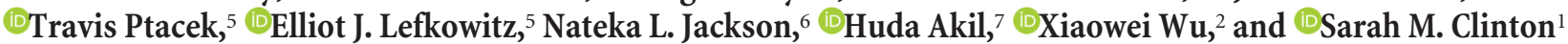 \\ ${ }^{1}$ School of Neuroscience, ${ }^{2}$ Department of Statistics, Virginia Polytechnic Institute and State University, Blacksburg, Virginia $24061,{ }^{3}$ Harry Perkins Institute \\ of Medical Research, University of Western Australia, Crawley WA 6009 Australia, ${ }^{4} \mathrm{MD} / \mathrm{PhD}$ Medical Scientist Training Program, ${ }^{5} \mathrm{Center}$ for Clinical and \\ Translational Science, Department of Microbiology, ${ }^{6}$ Department of Cellular, Developmental and Integrative Biology, University of Alabama at \\ Birmingham, Birmingham, Alabama 35233, and 7Molecular and Behavioral Neuroscience Institute, University of Michigan, Ann Arbor, Michigan 48109
}

There is growing evidence of abnormal epigenetic processes playing a role in the neurobiology of psychiatric disorders, although the precise nature of these anomalies remains largely unknown. To study neurobiological (including epigenetic) factors that influence emotionality, we use rats bred for distinct behavioral responses to novelty. Rats bred for low novelty response (low responder [LR]) exhibit high levels of anxiety- and depressive-like behavior compared with high novelty responder (HR) rats. Prior work revealed distinct limbic brain development in HR versus LR rats, including altered expression of genes involved in DNA methylation. This led us to hypothesize that DNA methylation differences in the developing brain drive the disparate HR/LR neurobehavioral phenotypes. Here we report altered DNA methylation markers (altered DNA methyltransferase protein levels and increased global DNA methylation levels) in the early postnatal amygdala of LR versus HR male rats. Next-generation sequencing methylome profiling identified numerous differentially methylated regions across the genome in the early postnatal HR/LR amygdala. We also contrasted methylation profiles of male HRs and LRs with a control rat strain that displays an intermediate behavioral phenotype relative to the HR/LR extremes; this revealed that the LR amygdalar methylome was abnormal, with the HR profile more closely resembling that of the control group. Finally, through two methylation manipulations in early life, we found that decreasing DNA methylation in the developing male and female amygdala improves adult anxiety- and depression-like behavior. These findings suggest that inborn DNA methylation differences play important roles in shaping brain development and lifelong emotional behavior.

Key words: amygdala; anxiety; depression; DNA methylation; neurodevelopment

Significance Statement

Epigenetic changes are biological mechanisms that regulate the expression and function of genes throughout the brain and body. DNA methylation, one type of epigenetic mechanism, is known to be altered in brains of psychiatric patients, which suggests a role for DNA methylation in the pathogenesis of psychiatric disorders, such as depression and anxiety. The present study examines brains of rats that display high versus low levels of anxiety- and depression-like behavior to investigate how neural DNA methylation levels differ in these animals and how such differences shape their emotional behavioral differences. Studying how epigenetic processes affect emotional behavior may improve our understanding of the neurobiology of psychiatric disorders and lead to improved treatments.

\section{Introduction}

Vulnerability to mental illness and ability to cope with stress are shaped by individual differences in temperament (Kagan et al., 1987; Rosenbaum et al., 2000; Biederman et al., 2001). Underpaper; S.M.C. designed research; C.R.M., M.E.G., L.T.F., R.K.S., J.L.C., and N.L.J. performed research; T.P., E.J.L., H.A., and X.W. contributed unpublished reagents/analytictools; C.R.M., X.W., and S.M.C. analyzed data; C.R.M. and S.M.C. wrote the paper. standing biological mechanisms that influence brain development, temperament, and vulnerability to emotional dysfunction is critical for elucidating the neurobiology of psychiatric illnesses,

This work was supported by National Institutes of Health Grants 4R00MH085859-02 to S.M.C., R01MH105447-01 to S.M.C., T90DE022736-04 to C.R.M., and T32GM008111 to M.E.G. We thank Dr. Jeremy J. Day for guidance in the MBD-seq studies.

The authors declare no competing financial interests.

Correspondence should be addressed to Sarah M. Clinton at sclinton@vt.edu.

https://doi.org/10.1523/JNEUROSCI.1157-15.2019

Copyright $\odot 2019$ the authors 
such as anxiety and depression, as well as for improving treatment strategies. To study neurodevelopmental factors that contribute to individual differences in emotionality, we use model rats that were selectively bred for distinct behavioral response to novelty. Rats bred for low response to novelty (low responders [LRs]) are highly inhibited in novel situations and display high levels of anxiety-like behavior, diminished sociability, low sexual motivation, and passive coping in response to stress compared with high novelty responder (HR) rats (Stead et al., 2006b; Stedenfeld et al., 2011; Cummings et al., 2013; Cohen et al., 2015). A valuable aspect of these lines is their phenotypic predictability (the ability to foretell whether a rat will grow up to display high or low novelty response from birth throughout life), which permits developmental neurobiological studies that were not previously possible. Our prior work with the bred HR and LR lines shows that the HR/LR behavioral traits emerge during the first weeks of life (Clinton et al., 2008, 2011), and that the traits are associated with distinct patterns of hippocampal development (Clinton et al., 2011; Turner et al., 2011). A more recent transcriptome study revealed marked gene expression differences in the HR/LR amygdala as well as in the hippocampus, including alterations of genes involved in epigenetic processes, such as DNA methylation (Simmons et al., 2012; McCoy et al., 2016b).

Growing evidence suggests that abnormalities in epigenetic processes (including DNA methylation) occur in the brains of individuals suffering major psychiatric disorders (Tsankova et al., 2007; Mill et al., 2008; Dempster et al., 2011; Sun et al., 2013). Many studies have shown how early-life experiences (e.g., exposure to stress or variation in maternal care) shape brain development and emotional behavior through epigenetic mechanisms (McGowan et al., 2009; Murgatroyd et al., 2009; Roth et al., 2009; LaSalle, 2011; Naumova et al., 2012; Bedrosian et al., 2018). However, far less is known of inborn interindividual epigenetic differences that likely also play important roles in driving psychiatric disease susceptibility and pathology (Rakyan and Beck, 2006; Bock et al., 2008; Richards, 2008; Zhang et al., 2010). Furthermore, another important knowledge gap in the field relates to the developmental timeline when such epigenetic changes unfold because most studies in psychiatric patients and model animals relevant to psychopathology focus on changes in the adult brain.

Prior transcriptome studies in our laboratory uncovered vast gene expression differences in the developing hippocampus and amygdala of HR versus LR rats (Clinton et al., 2011; McCoy et al., $2016 \mathrm{~b})$. These gene changes included molecules involved in epigenetic processes, particularly DNA methylation, thereby pointing to a regulatory mechanism that could underlie widespread HR/LR differences in gene expression and ultimately behavior. Based on these findings, we hypothesized that (1) HR/LR differences in neural DNA methylation patterns constitute a key phenotype-driving molecular mechanism; and (2) manipulating methylation in the developing brain (to recapitulate an HR-like methylation pattern) can modulate adult emotional behavior. To test this, the present study examined DNA methyltransferase (DNMT) protein levels and global DNA methylation (5methylcytosine) levels in the early postnatal HR/LR brain. Nextgeneration sequencing was then used to interrogate gene-specific HR/LR methylation differences. Based on findings of increased DNA methylation levels in the developing amygdala of LR versus HR pups, we tested whether decreasing DNA methylation in the early postnatal amygdala (via a dietary manipulation or siRNAmediated approach) could lead to improved anxiety- and/or depression-like behavior in adulthood. Our results suggest that inborn DNA methylation differences in the developing brain in- fluence lifelong emotional behavior, which may shed light on neural mechanisms relevant to the neurobiology of emotional disorders and new potential treatments.

\section{Materials and Methods}

\section{Animals}

Rats were obtained from our in-house colony where the HR/LR bred lines were rederived using a breeding strategy described in our original publication with the bred HR/LR rats (Stead et al., 2006b). The present experiments used HR/LR male and female rats from the fourth, fifth, sixth, and eighth generations of our colony. Because HR/LR rats represent extreme behavioral phenotypes, one of the current experiments includes a control group with intermediate novelty responder (IR) rats. These IR rats were created by cross-breeding the HR/LR lines and have been shown to display intermediate novelty-induced activity and anxiety-like behavior relative to HR/LR counterparts (Flagel et al., 2014). For all experiments, housing and testing facilities were maintained at $21^{\circ} \mathrm{C}-23^{\circ} \mathrm{C}$ and $50 \%-55 \%$ humidity; rats were pair-housed in a $12: 12$ light-dark cycle (lights on/off at 6:00 A.M./6:00 P.M.). Studies were approved by the local Institutional Animal Care and Use Committee, and the work was performed in accordance with the National Institutes of Health (2011) and National Research Council (2011) guidelines on animal research.

DNMT expression and global DNA methylation (5-methylcytosine) levels in early postnatal $H R$ versus LR amygdala and hippocampus At birth, HR and LR litters were culled to 6 male and 6 female pups to control for litter size and gender composition. Offspring were killed by rapid decapitation to harvest brains at three early postnatal time points: postnatal day (P) 7, P14, and $\mathrm{P} 21$ ( $n=16$ pups/phenotype/time point). To avoid "litter effects," experimental groups were comprised of animals from independent litters. Only male offspring were studied in the experiments described here.

Brains were removed, flash frozen in isopentane, cooled to $-30^{\circ} \mathrm{C}$ on dry ice, and then stored at $-80^{\circ} \mathrm{C}$ until further use. Brains were sectioned on a cryostat at $-10^{\circ} \mathrm{C}$ to $-12^{\circ} \mathrm{C}$, with alternating sections of 20 and 300 $\mu \mathrm{m}$ collected. The $20 \mu \mathrm{m}$ sections were stained with cresyl violet to identify target anatomical regions in the $300 \mu \mathrm{m}$ sections. Portions of the hippocampus and amygdala were removed from the $300-\mu \mathrm{m}$-thick sections using a surgical scalpel (\#11) and then homogenized. One set of samples was used to isolate protein (EpiQuik Nuclear Extraction kit, Epigentek, catalog \#OP-0002-1; $n=8$ /group). Extracted protein concentration was quantified using a BCA protein assay (Thermo Fisher Scientific), and samples were used in EpiQuik DNMT1, DNMT3a, and DNMT3b protein assays (Epigentek, catalog \#P-3011, P-3012, and P-3013, respectively) according to the manufacturer's instructions. Separate samples were used to isolate DNA (DNeasy, QIAGEN; $n=$ 8/group). DNA was quantified using a Nanodrop ND-1000, stored at $-20^{\circ} \mathrm{C}$, and later used to assess levels of 5-methylcytosine (DNA methylation) and 5-hydroxymethylcytosine (DNA hydroxymethylation). The MethylFlash Methylated DNA Quantification Kit (Colorimetric; Epigentek) measured global methylation, and the Hydroxymethylated DNA Quantification kit (Colorimetric; Epigentek) assessed global hydroxymethylation per the manufacturer's instructions and as we previously described (Simmons et al., 2013).

\section{DNA methylation profiling in HR versus LR early postnatal} amygdala using next-generation sequencing

To obtain a more detailed understanding of DNA methylation differences in the early postnatal (P7) HR versus LR amygdala, we performed an experiment using methylated DNA capture coupled with nextgeneration sequencing (MethylCap-Seq). Moreover, as an additional control, these experiments also included samples from IR rats, which display an intermediate behavioral phenotype relative to the selected HR/LR lines (Flagel et al., 2014).

Methylated DNA was captured using a MethylMiner DNA enrichment kit (Applied Biosystems, ME10025) according to the manufacturer's recommended protocol. DNA samples from P7 male HR, LR, and IR amygdala ( $n=4$ /group) were sheared by sonication using a Bioruptor 
Pico (Diagenode, B01060001). The sonication cycle consisted of $15 \mathrm{~s}$ on $/ 45 \mathrm{~s}$ off for 7 total cycles to generate fragments of (on average) $450 \mathrm{bp}$ for the methylated DNA enrichment protocol as previously described (Brinkman et al., 2010). Methylated DNA fragments were captured with a methyl-binding domain 2 (MBD2) protein coupled to paramagnetic Dynabeads M-280 Streptavidin. Starting material for the enrichment protocol was $1 \mu \mathrm{g}$ of sonicated DNA, and captured fragments were eluted using $2500 \mathrm{~mm} \mathrm{NaCl}$ per the manufacturer's instructions and as described previously (Brinkman et al., 2010). Captured material was purified using QIAquick PCR purification spin columns (QIAGEN) and quantified using Quant-it high sensitivity DNA Assay Kit (Invitrogen, Q-33120) and a 2100 Bioanalyzer high sensitivity chip kit (Agilent Technologies). Samples were sent to HudsonAlpha Genomic Services Laboratory (Huntsville, $\mathrm{AL}$; http://gsl.hudsonalpha.org) for next-generation sequencing. Barcoded DNA fragment libraries were created, checked for quality, and quantified with the Kapa Library Quant Kit (Kapa Biosystems). Afterward, each library was used for high-throughput sequencing on a HiSeq 2000 (Illumina) with a minimum of 25 million reads total 50 bp single-end reads per sample. We sequenced four biological replicates per group as well as an input (noncaptured) control for normalization.

To ensure that MBD2 protein capture resulted in specific enrichment of methylated DNA in our laboratory, we performed control reactions. In these reactions, gDNA was spiked with synthetic methylated and nonmethylated DNA fragments (1 pg each, Methyl Miner kit, Invitrogen) before immunoprecipitation with recombinant MBD2. We performed PCR with primers for these synthetic fragments within the methylated and nonmethylated DNA capture, using both the captured (MBD2bound) and unbound fractions. Our results demonstrated the presence of methylated DNA fragments in the captured sample and the absence of methylated DNA in the unbound fraction.

We imported sequencing data files into Galaxy (http://g2.bx.psu.edu), an online data analysis system that facilitates large-scale genome analyses (Giardine et al., 2005; Blankenberg et al., 2010; Goecks et al., 2010). Raw single-end sequenced reads were quality controlled and filtered for read quality (FastQC toolkit, Galaxy). Sample reads were mapped onto the rat genome reference sequence (Rn5 assembly) using the high-performance alignment software Bowtie (http://bowtie-bio.sourceforge.net/index. shtml). We have archived this dataset in NCBI SRA. Overall, we obtained an average of 37.4 million mapped single-end reads from Methyl-Cap samples. Data analysis was conducted using MEDIPS, a coverage analysis $\mathrm{R}$ program designed for enrichment studies (Lienhard et al., 2014). MEDIPS compares input and enriched samples to find the signal density (reads per kilobase per million) and then contrasts these measures among experimental groups. We have archived this dataset in NCBI SRA (ID: PRJNA483463).

Our first analysis focused on comparing differentially methylated regions (DMRs) between HR and LR samples, using a nominal level of 0.05 with Bonferroni multiple test corrections. These liberal statistical parameters were set to survey global patterns of methylation differences. Methylation patterns were examined within several genomic features, including the cis-regulatory regions of genes, which enabled us to evaluate distinct HR/LR methylation within 10 kilobases $(\mathrm{kb})$ upstream and downstream of a gene and across the gene body. Genes that showed differential methylation within the gene body or $10 \mathrm{~kb}$ upstream of the transcription start site were also examined with Kyoto Encyclopedia of Genes and Genomes (KEGG) analysis using Enrichr (Kuleshov et al., 2016).

\section{Comparing DNA methylation patterns in the $H R, L R$,}

\section{and IR amygdala}

Our next phase of analysis sought to compare methylation patterns in HR, LR, and IR amygdala to determine whether methylation differences in $H R$ versus LR samples predominantly reflect an aberration of one group or the other compared with "normal" IRs (i.e., whether LRs exhibit abnormally high methylation levels or whether HRs exhibit abnormally low levels relative to an IR control). We examined methylation peak values (corrected against input in reads per kilobase per million) for HR, LR, and IR rats, focusing on the 1881 peaks that were identified as differentially methylated between HRs and LRs in the first analysis phase.
Using the averaged HR, LR, and IR methylation profiles, we first examined correlations between (1) HR and IR profiles and (2) LR and IR profiles. These results were used to create scatterplots of each methylation site and calculate the overall correlation between IR and either HR or LR. We then focused on DMRs that previously differed between HR/LR rats and asked whether this new analysis found differences between HR and IR and/or between LR and IR. This approach allowed us to ask whether IR methylation patterns are more similar to HR or LR methylation patterns, or whether IRs display a unique intermediate pattern of DNA methylation relative to HRs and LRs. The null hypothesis was that the outcomes would show equal frequency of HR-like and LR-like methylated sites. To address this hypothesis, we again used a threshold of $p<$ 0.05 when comparing DMRs between HRs and LRs; this yielded a total of $934 \mathrm{HR} / \mathrm{LR}$ DMRs within or surrounding genes to then be examined in the HR/IR/LR analysis. We used McNemar's $\chi^{2}$ test using a $2 \times 2$ contingency table of dichotomous data for matched pairs of subjects to determine whether row and column marginal frequencies are equal (whether there is "marginal homogeneity").

\section{Examining behavioral effects of perinatal dietary methyl donor} depletion in $H R / L R$ offspring

Given the observed DNA methylation differences in the early postnatal HR versus LR brain, a pivotal next question was as follows: what role do these methylation differences play in shaping the disparate HR/LR behavioral phenotypes? We hypothesized that LRs' elevated DNA methylation levels in the early postnatal amygdala (relative to HRs) contribute to differences in limbic brain development and ultimately their disparate anxiety-/depression-like behaviors. We first tested this hypothesis by manipulating methyl donor availability in perinatal HR/LR rats via manipulation of dietary methyl donor content of pregnant/lactating rat HR or LR mothers. The experiment was conducted in two phases: the first phase in LR dams and the second phase in HR dams. Adult male/female LR or HR pairs were mated to produce offspring for each study. Beginning at gestational day 17 (G17), females were randomly assigned to receive (1) a control diet of standard rat chow (CON; $n=11$ LR dams, $n=6$ HR dams); or (2) chow that was $90 \%$ depleted of methyl donors (DEP; $n=8$ LR dams, $n=5$ HR dams). The CON dams received commercially made semisynthetic L-amino acid-complete rodent diet no. A10021 (Research Diets). The DEP group received an L-amino aciddefined rodent diet lacking $90 \%$ of the normal requirements of choline, folate, and methionine (diet no. A04062402, Research Diets). At birth, litters were culled to 6 male/ 6 female pups. Dams continued to receive assigned diets through P21 when pups were weaned. We chose to initiate the maternal diet in late gestation (G17), in part to avoid gross birth defects that might occur due to methyl donor depletion from conception onward. In addition, we felt another advantage of the G17 time point was that it coincides with a time when limbic system predominates, whereas other major neural systems (e.g., motor and sensory systems) are largely established (Moore et al., 2006).

Maternal behavior of dams exposed to CON and DEP diets. To determine whether the methyl diet manipulation affected dams' behavior, we observed maternal behavior in CON and DEP females from P1 to P14 using a previously published protocol (Clinton et al., 2010). Each cage was observed twice daily: once during the light phase (at 10:00 A.M.) and once during the dark phase (at 9:00 P.M.). Each observation period (lasting $45 \mathrm{~min}$ to $1 \mathrm{~h}$ ) consisted of a series of $105 \mathrm{~s}$ "snapshot" observations for each cage, which were taken $\sim 5$ min apart. During a "snapshot" observation, a checklist was used to note which behaviors were being observed. The behaviors noted were as follows: (1) mother licking or grooming a pup; (2) mother resting away from litter; (3) mother passive nursing pups; and (4) mother arched-back nursing pups. Passive nursing was defined as the mother lying on her side or back and nursing any number of pups. Arched-back nursing was classified as the mother arched over any number of nursing pups with her legs extended. By the end of the 14 observation days, each cage had accumulated 280 observations ( 10 observations per session $\times 2$ sessions each day $\times 14$ total days).

Behavioral test battery in offspring exposed to CON and DEP diets. On P21, male and female offspring of the CON and DEP dams were weaned. These offspring will be referred to as $\mathrm{pDEP}$ or $\mathrm{pCON}$ to designate peri- 
natal exposure to the methyl donor-deprived or control maternal diets, respectively. Offspring were housed 2 or 3 per cage (separated by sex and experimental condition) and raised in standard housing (including standard rat chow) until they reached adulthood $(\mathrm{P} 70+)$. In the LR experimental phase, adult male and female pDEP and pCON offspring $(n=$ 14-16 per group) embarked on a behavioral test battery consisting of the Open Field Test (OFT), the Elevated Plus Maze (EPM), a Social Interaction Test, Sucrose Preference Test, and the Forced Swim Test (FST). In the HR experimental phase, adult HR offspring (HR-pCON, $n=12$ males, 13 females; HR-pDEP, $n=6$ males, 5 females) were evaluated in the same tests. All animals were subjected to the full test battery in this test order, with 1-2 d rest between tests. Males and females were tested on separate days, and all tests were performed between 8:00 A.M. and 12:30 P.M. under dim lighting (30 lux). Behavioral tests were conducted as previously described (Nam et al., 2014; Cohen et al., 2015). For all tests, the Ethovision XT 8.0 video tracking software (Noldus) was used to video record and analyze behavior.

OFT. The OFT was conducted in a black Plexiglas box $(100 \times 100 \times 50$ $\mathrm{cm}$ ). The rat was placed into one corner of the apparatus at the start of the test and permitted to explore for $5 \mathrm{~min}$. The latency to enter the center of the OF, the amount of time spent, and distance traveled in the center, periphery, and corners of the apparatus were quantified. A trained observer blinded to experimental conditions manually assessed grooming and rearing behavior using a component of the Ethovision software.

$E P M$. The EPM apparatus was constructed of black Plexiglas with four elevated arms ( $70 \mathrm{~cm}$ from the floor, $45 \mathrm{~cm}$ long, $12 \mathrm{~cm}$ wide) arranged in a cross. Two opposite arms were open (lighting at 30 lux), and the other two arms were enclosed by $45-\mathrm{cm}$-high walls, which limited lighting to 3-5 lux. A rat was placed in the center square of the EPM facing a closed arm at the test start, and it was then allowed to freely explore for $5 \mathrm{~min}$. Ethovision was used to monitor latency to enter the open arms, time spent, and frequency to visit the open arms, closed arms, and center square.

Social interaction. The Social Interaction Test was conducted as previously described (Nam et al., 2014) using a 2 d procedure where experimental animals spent $10 \mathrm{~min}$ per day in a clear Plexiglas chamber $(45 \times$ $45 \times 60 \mathrm{~cm}$ Noldus PhenoTyper Box) containing a smaller "interaction box" $(10 \times 10 \times 8 \mathrm{~cm})$ that held a novel stimulus rat. On test day 1 , a novel interactor of the same sex was placed in the interaction box shortly before the experimental rat was introduced. On test day 2, a novel interactor of the opposite sex was placed in the interaction box shortly before the experimental rat was introduced. The interaction box allowed for the experimental animal to approach, see, and smell the interactor without the potential for physical contact. To assess experimental animals' level of social interaction, we defined specific zones in the test chamber as either "interaction" or "avoidance" zones. Thus, a 2-cm-wide zone around the "interaction box" was designated as the "interaction zone," and a $10 \times 10$ $\mathrm{cm}$ zone opposite to the "interaction box" was considered the avoidance zone. Time spent within each zone and number of visits to each zone during the two sessions was quantified by Ethovision. A blinded, trained observer scored grooming behavior and stress-induced defecation as secondary measures of rodent anxiety.

Sucrose preference. At the start of the dark cycle, each cage was provided with two bottles with one bottle (randomly selected) filled with $0.5 \%$ sucrose solution. Bottles were then weighed at three later time points ( 1 , 2 , and $12 \mathrm{~h}$ after being exposed to the sucrose solution); sucrose preference was determined by the percent sucrose solution intake per total volume consumed.

FST. Porsolt's FST was performed as previously described (Nam et al., 2014 ) with 30 -cm-deep $25^{\circ} \mathrm{C}$ water in Plexiglas containers $(45 \mathrm{~cm}$ high $\times$ $20 \mathrm{~cm}$ diameter). On FST day 1 , rats were placed (1/cylinder) into the water for $15 \mathrm{~min}$; $24 \mathrm{~h}$ later, they were returned to the water-filled cylinder and tested for another $5 \mathrm{~min}$. Water was changed after every swim session so that every rat was swimming in clean water. Ethovision was used to score rats' immobility on each test day. We focused on the immobility measure because it is classically considered to be an indicator of behavioral despair and depressive-like behavior (Porsolt et al., 1977), and it can be clearly defined from active coping measures, such as swimming and climbing (Cryan et al., 2005).
Assessing behavioral consequences of transient siRNA-mediated

DNMT3b knockdown in the early postnatal amygdala

Based on our findings of higher levels of global methylation and the DNMT, DNMT3b, in the early postnatal amygdala of LR versus HR rats, we hypothesized that suppressing DNMT3b in the early postnatal amygdala would induce lasting changes in emotional behavior, potentially leading to a low anxiety/depression-like HR phenotype. To test this hypothesis, we infused an Accell siRNA designed to target and transiently suppress the expression of Dnmt3b (or control siRNA) into the amygdala of P10 IR pups. We chose to use IR rats for this experiment because they show a predictable intermediate behavioral phenotype. Although it would have been ideal to perform the siRNA injections at an earlier time point (i.e., at or before P7), we felt that stereotaxic surgery in rats younger than P10 was pushing the limits of technical feasibility and likely animal viability. Thus, we chose the P10 time point because it falls within the timeframe when we observed HR/LR DNA methylation differences in the amygdala (P7-P14). Previously published work with these siRNAs demonstrated effective knockdown for 2-7 d after treatment (Nakajima et al., 2012; Mitchnick et al., 2015). As noted, here, we have included a subset our Dnmt3b siRNA and control siRNA treated rats for death $4 \mathrm{~d}$ after treatment to confirm Dnmt $3 b$ knockdown.

Adult male and female rats were mated to produce offspring for the surgeries. At birth, litters were culled to 6 male/ 6 female pups. On P10, using a within-litter design, 1 or 2 male and 1 or 2 female pups per litter (total $n=14$ or 15/condition/sex) received bilateral injections of Accell SMARTpool siRNA targeting Dnmt3b (E-103388-03-0005 Dharmacon) or a negative control siRNA (K-005000-G1-02) into the amygdala (anteroposterior $-2.2 \mathrm{~mm}$, mediolateral $\pm 4.1 \mathrm{~mm}$, dorsoventral $7 \mathrm{~mm}$ relative to bregma). Accell siRNA aliquots were diluted in Accell siRNA resuspension buffer (working concentration $15 \mu \mathrm{M}$ ), stored at $-20^{\circ} \mathrm{C}$; and fresh aliquots were used daily. Animals were anesthetized with isoflurane, and $0.5 \mu \mathrm{l}$ of siRNA was injected ( $0.1 \mu \mathrm{l}$ per minute over $5 \mathrm{~min})$. Following surgery, pups were returned to their mothers and remained undisturbed until weaning at P21. Offspring were monitored daily to assess potential negative outcomes following surgery; all dams resumed sufficient maternal care of pups following surgeries, and no offspring were rejected by the dam. A subset of the pups that underwent stereotaxic surgery were killed $4 \mathrm{~d}$ after surgery to harvest brain tissue to confirm siRNA-mediated knockdown of Dnmt3b expression in the amygdala of Dnmt3b siRNA-treated versus control siRNA-treated pups $(n=5$ per condition). The remaining pups (control siRNA, $n=14$ males and 15 females; Dnmt3b siRNA, $n=15$ males and 14 females) were weaned on P21, housed 2 or 3 per cage (separated by sex and experimental condition), and raised to adulthood to be evaluated in the behavioral test battery described above. To avoid litter effects, experimental groups contained no more than one pup per sex from a particular litter and a within-litter design was implemented.

\section{Experimental design and statistical analyses}

Across all studies, age and sex of animals were considered separate factors and therefore not combined in analysis. Because many of our studies involved developmental manipulations, potential litter effects were considered and addressed as noted above. Animal numbers for each study are outlined in Materials and Methods. For all experiments, researchers were blinded to animals' experimental groups at the time of data collection.

Data from the next-generation sequencing analyses are described in Materials and Methods. All other data were analyzed using Prism Software (version 6.0 for Windows, GraphPad Software; www.graphpad. com). In these instances, data were verified to be normally distributed using the D'Agostino and Pearson omnibus normality test before proceeding with subsequent analyses. If data were not normally distributed, an appropriate nonparametric test was chosen. For all $t$ tests or ANOVAs (or nonparametric equivalent), the differences were considered significant at $p<0.05$, and results are presented as mean \pm SEM.

Two-tailed Student's $t$ tests were used to compare DNA methylation markers in HR/LR brain regions (independent variable being HR/LR phenotype). The perinatal dietary methyl donor depletion study was split into an LR phase and HR phase where dams were fed DEP or CON; 
consequently, we separately examined the effect of diet manipulation on each phenotype. Maternal behaviors were analyzed by two-way ANOVA (independent variables: DEP/CON diet and postpartum day). Adult offspring of the mothers $(\mathrm{pDEP} / \mathrm{pCON}$ denote perinatal exposure to the DEP/CON conditions) were evaluated in a behavioral test battery. Given the substantial weight differences between $\mathrm{pDEP} / \mathrm{pCON}$, we used linear models to consider multiple factors in behavioral measures examined. Thus, offspring behavioral data from the OFT, EPM, and FST were treated as responses in the linear model, with perinatal methyl donor condition, sex, and weight as independent variables. Social interaction and sucrose preference data were analyzed in the same way with responses being time spent with the opposite sex/time spent with the same sex and sucrose preference in later time point/sucrose preference in earlier time point, respectively.

The early-life siRNA-mediated Dnmt $3 b$ knockdown experiment in IR rats examined the lasting behavioral effects following transient Dnmt3b suppression in the early postnatal amygdala. Behavioral data from adult male and female offspring exposed to early-life Dnmt3b manipulation were analyzed separately as substantial sex differences are known to exist in behavioral measures. Here, two-tailed Student's $t$ test was performed for each behavioral measure to compare treatments within each sex. For social interaction and sucrose preference, the data were analyzed by twoway ANOVA (independent variables): (1) siRNA treatment; and (2) for social interaction, time spent with either novel animal of same or opposite sex; and for sucrose preference, time point following sucrose solution presentation).

\section{Results \\ DNA methylation markers in the early postnatal HR/LR amygdala and hippocampus}

Given our previous findings of altered Dnmt1 mRNA levels in the developing HR/LR limbic brain (Simmons et al., 2012), coupled with our interest in epigenetic mechanisms that potentially drive HR/LR differences in neural gene expression and behavior (Clinton et al., 2011; McCoy et al., 2016b), this first experiment evaluated multiple DNA methylation markers in the HR/LR amygdala and hippocampus at three early postnatal time points: P7, P14, and P21. We focused on these two brain regions and particular time points because a previous transcriptome profiling experiment pointed to dramatic gene expression changes in both areas during the first 1-2 weeks of life (McCoy et al., 2016b). In each region, we measured protein levels of the major DNMTs (DNMT-1,-3a, and -3b) as well as 5-methylcytosine (an indicator of overall DNA methylation) and 5-hydroxymethylation (an indicator of DNA hydroxymethylation, which occurs when Teneleven translocation [Tet] methylcytosine dioxygenases add a hydroxyl group to methylated cytosines in DNA) (Moore et al., 2013).

In the P7 amygdala, LR rats (compared with HRs) showed increased DNMT1 $\left(t_{(10)}=2.638, p=0.0248\right.$; unpaired $t$ test $)$; decreased DNMT3a $\left(t_{(9)}=2.624, p=0.0276\right.$; unpaired $t$ test $)$; increased DNMT3b $\left(t_{(12)}=2.310, p=0.0395\right.$; unpaired $t$ test $)$; and increased global DNA methylation $\left(t_{(12)}=5.994, p<0.0001\right.$; unpaired $t$ test; Fig. 1A). LRs also showed increased DNMT1 protein levels in the P14 amygdala compared with their HR counterparts $\left(t_{(7.988)}=2.831, p=0.0222\right.$; Welch's corrected unpaired $t$ test). There were no HR/LR differences in these DNA markers in the P21 amygdala. In the hippocampus, we found no differences in DNMT1-1, $-3 \mathrm{a}$, or $-3 \mathrm{~b}$ protein levels and no global DNA methylation differences at any age examined (Fig. 1B).

Next, we asked whether global hydroxymethylation levels were altered. In amygdala, there was an effect of age $\left(F_{(2,42)}=\right.$ 5.366, $p=0.0084$; two-way ANOVA), where global hydroxymethylation levels were not found to be different in post hoc analysis, at P7 $(\mathrm{HR}=0.0158 \pm 0.0018, n=8 ; \mathrm{LR}=0.0164 \pm 0.0021$ $n=8), \mathrm{P} 14(\mathrm{HR}=0.0297 \pm 0.0005 n=8, \mathrm{LR}=0.0245 \pm 0.0037$, $n=8)$, and P21 $(\mathrm{HR}=0.02136 \pm 0.0033, n=8, \mathrm{LR}=0.0240 \pm$ $0.0040, n=8)$. There were no differences in global hydroxymethylation at $\mathrm{P} 7(\mathrm{HR}=0.0169 \pm 0.0025, n=8 ; \mathrm{LR}=0.0132 \pm$ $0.0014 n=8), \mathrm{P} 14(\mathrm{HR}=0.0185 \pm 0.0027, n=8 ; \mathrm{LR}=0.0143 \pm$ $0.0016 n=8)$, or P21 ( HR $=0.01126 \pm 0.0016, n=7$; LR $=$ $0.0162 \pm 0.0021 n=7)$.

\section{Mapping the methylome in the developing LR versus HR amygdala}

To delve more deeply into HR/LR DNA methylation differences in the early postnatal amygdala, we used next-generation sequencing technology to map and compare the methylome of the HR versus LR P7 amygdala. Figure $2 A$ illustrates the methylome of $\mathrm{HR}$ versus LR amygdala using a circle plot, which provides a genome-wide chromosome-by-chromosome view of average methylation profile of HR (green, outer track) and LR (red, inner track). Using a MethylCap-Seq enrichment approach, we sequenced methylated DNA fragments with the resolution of a few hundred bases pairs and used MEDIPS to assess DMRs between HR/LR. These analyses yielded 1881 DMRs with 1566 that are hypermethylated in LR compared with HR samples and 315 hypomethylated in LR versus HR. These results point to a preponderance of hypermethylated sites in LR compared with HR samples, which is consistent with our finding of increased global methylation in the P7 LR versus HR amygdala.

Our next phase of analysis filtered the data based on the distance of DMRs from genes (see illustration in Fig. 2B). We evaluated the distribution of DMRs in the LR versus HR amygdala broken down location proximal to genes as well as their hypermethylation or hypomethylation status (Fig. 2C). Using genes with DMRs within the gene body or $10 \mathrm{~kb}$ upstream of the gene, we performed KEGG analysis to find functional pathways enriched with genes that displayed LR hypermethylation (compared with HR; Fig. 2D; threshold: $p<0.05$ ). The top enriched pathway was Ras-related protein 1 (Rap1) signaling pathway, which receives signals through membrane receptors including ionotropic glutamate NMDARs and G-protein-coupled receptors to activate multiple downstream intracellular signaling pathways, such as activation of mitogen-activated protein kinases or phosphoinositide 3-kinase-protein kinase B (PI3K-Akt). Another highly enriched pathway pertained to long term potentiation, which includes functions, such as regulation of the glutamate synapse through AMPARs.

\section{Comparing DNA methylation profiles in $\mathrm{HR}, \mathrm{LR}$, and the intermediate IR rat}

The selectively bred HR and LR lines exhibit numerous neurobiological and behavioral differences compared with each other, but the question is often raised whether one of the phenotypes in particular differs from "normal" or whether each line represents an extreme that deviates from normal. In the context of the present study, a reasonable question is whether LR pups show abnormally high levels of DNA methylation relative to "normal," or whether HR pups display abnormally low DNA methylation levels in the early postnatal amygdala. Thus, we conducted an additional experiment to compare HR/LR methylome profiles with that of IR rats, which are created by cross-breeding between the HR/LR lines and known to exhibit an intermediate behavioral phenotype relative to extremes of the bred HR/LR lines (Flagel et al., 2014). Here we asked whether the DNA methylation profile in the P7 amygdala of IR rats more closely resembles an "HR-like" 


\section{A Amygdala}
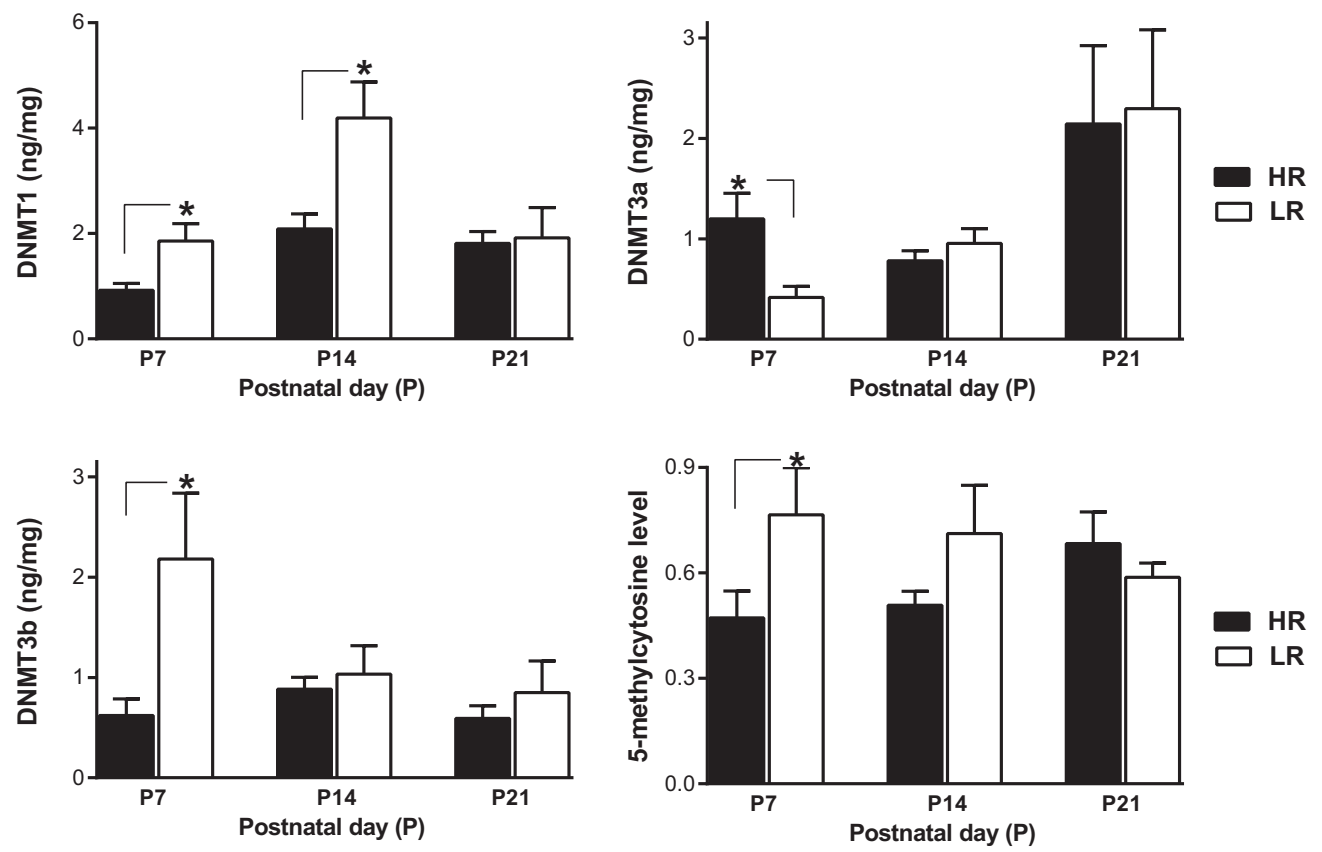

B

Hippocampus
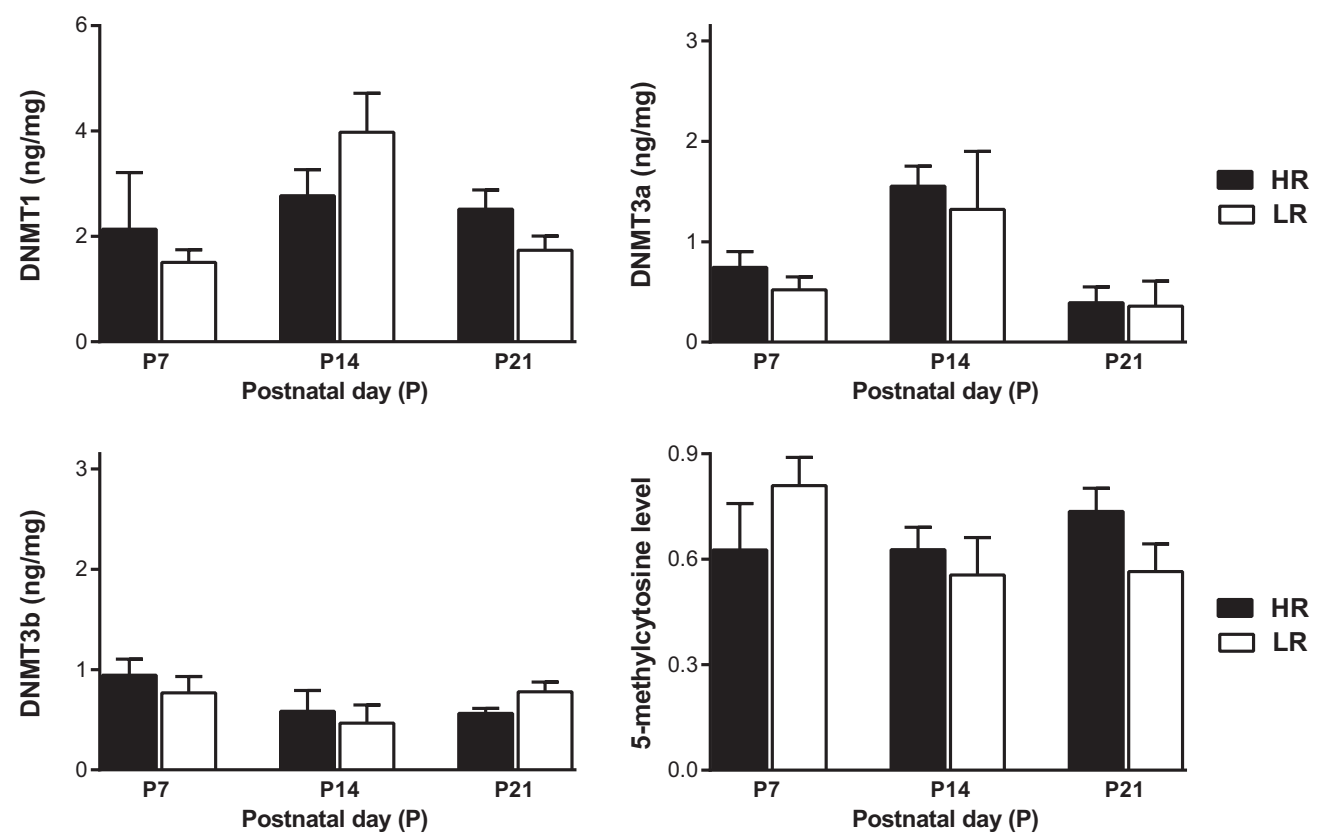

Figure 1. DNMT protein levels and global DNA methylation levels in the early postnatal HR versus $L R$ amygdala and hippocampus. $A$, LR rats exhibited higher levels of DNA methylation (5-methylcytosine; $n=5-7)$ and DNMT1 $(n=5-7)$, DNMT3a $(n=5-7)$, and DNMT3b $(n=7)$ protein levels in the amygdala at postnatal day (P)7 compared with HRs, with little to no difference at the later ages examined (P14 and P21). $\boldsymbol{B}$, In the hippocampus, there were no HR/LR differences in DNA methylation markers (global DNA methylation, $n=5 ;$ DNMT1, $n=5-7 ;$ DNMT3a, $n=$ $4-7$; DNMT3b, $n=7$ ) at any age examined. There were no HR/LR hydroxymethylation differences in the amygdala or hippocampus at any age examined. ${ }^{*} p<0.05$ for HR versus $L R$ comparisons. All sample sizes are per age and phenotype.

or "LR-like" profile, or whether they show an overall intermediate methylation level relative to these two groups.

To compare HR, LR, and IR samples, we focused on sites that showed differential methylation in HR/LR samples and created a scatterplot to correlate the averaged methylation levels for HR, LR, and IR at these sites (Fig. 3). The scatter plots indicate a more linear relationship between $\mathrm{HR}$ and IR than between HR/LR and between LR/IR (Fig. 3). Next, we calculated the overall correlations represented in Figure 3 between the DNA methylation profiles of each group. Here we again found that HR/IR profiles are more similar $(r=0.966)$ than LR/IR $(r=0.774)$ or HR/LR $(r=$ 0.684 ). Last, we used McNemar's $\chi^{2}$ test to compare the number 

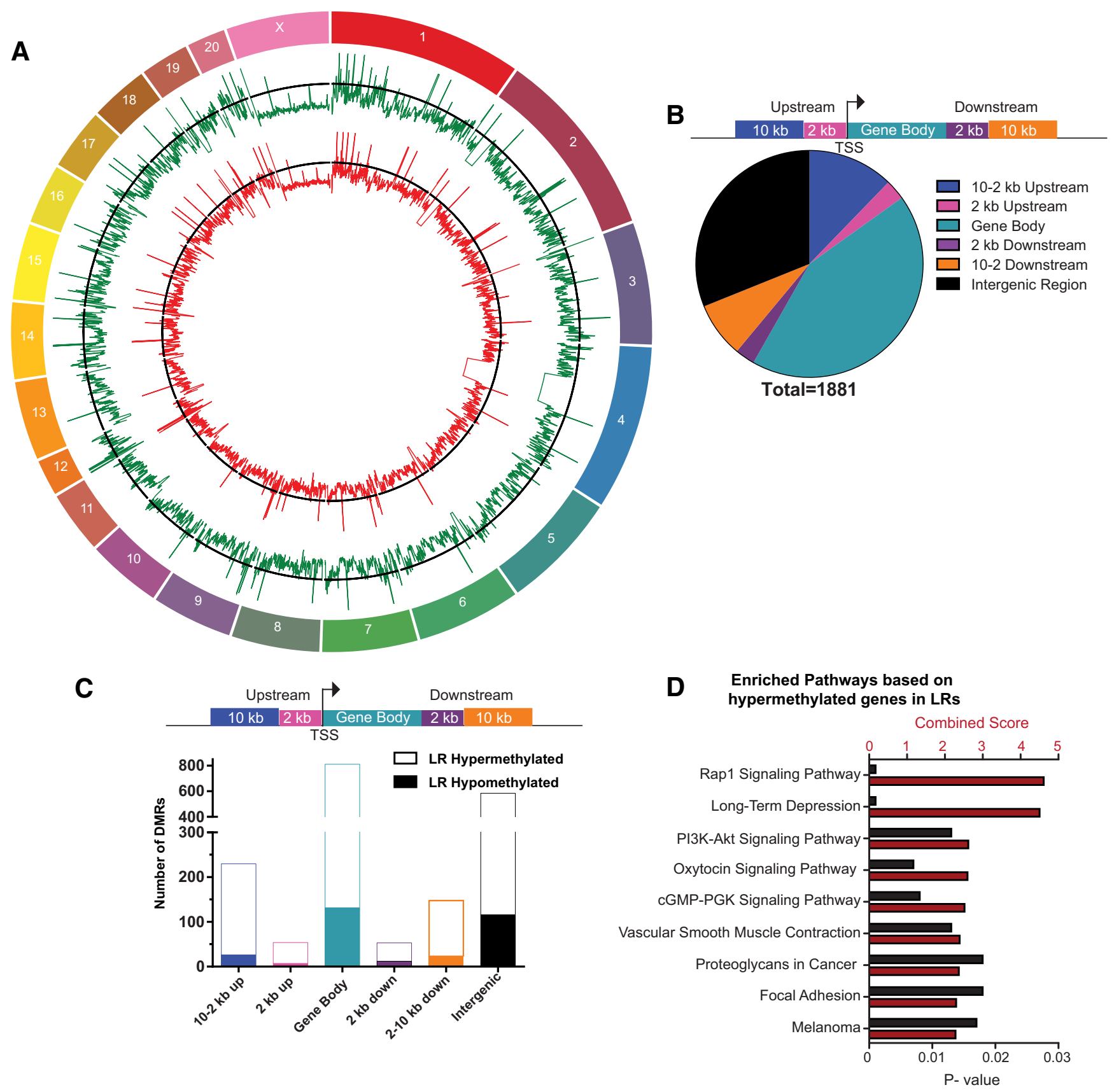

Figure 2. The distribution of HR versus LR methylation differences across standard genomic structures. A, Using MEDIPS (statistically filtering using edgeR, $p<0.05$ with Bonferroni correction), 1881 DMRs were identified in the HR/LR postnatal day 7 amygdala samples. The genome-wide distribution of methylated sites is shown through a circle plot with average HR (green) and average LR (red) methylation. $\boldsymbol{B}$, Diagram represents standard genomic regions where we examined potential HR/LR methylation difference. Pie chart represents the number of DMRs upstream and downstream of genes, within gene bodies and in intergenic regions. C, DMRs were grouped to show the number of sites that were hypomethylated in LR versus $H R$ samples (solid bars) and those that were hypermethylated in LR versus HR (open bars) across different genomic regions. D, KEGG analysis was used to identify functional pathways enriched with genes that were hypermethylated in LR versus HR amygdala samples. Here, combined enrichment score is shown on the top $x$-axis and is represented by red bars. The $p$ value of each pathway enrichment is shown on the bottom $x$-axis and is represented by black bars. All sample sizes were 4 per group. TSS = transcriptional start site.

of DMRs between either HR/IR or LR/IR within all HR/LR DMRs. Here, we found (1) 172 sites that were significantly different between LR/IR but not between HR/IR, and (2) 122 sites that were differentially methylated between HR/IR but not between LR/IR. This analysis revealed a significant difference in number of sites ( $p=0.004267$; Table 1$)$, indicating that that the IR methylation profile shows more similarity to HR than LR. Overall, these two independent analytical approaches (correlation analysis and $\chi^{2}$ analysis) both pointed to greater similarity between HR and IR DNA methylation profiles, thereby suggesting that LRs exhibit a unique (putatively "abnormal") DNA methylation profile.

Manipulating dietary methyl donors in LR dams improves adult LR offspring's anxiety- and depression-like behavior To begin to examine how LRs' heightened DNA methylation levels in the early postnatal brain contributes to their adult behavioral phenotype, we sought to decrease methylation in developing LRs by feeding LR dams a diet severely lacking methyl donors. We chose this maternal diet manipulation approach, 

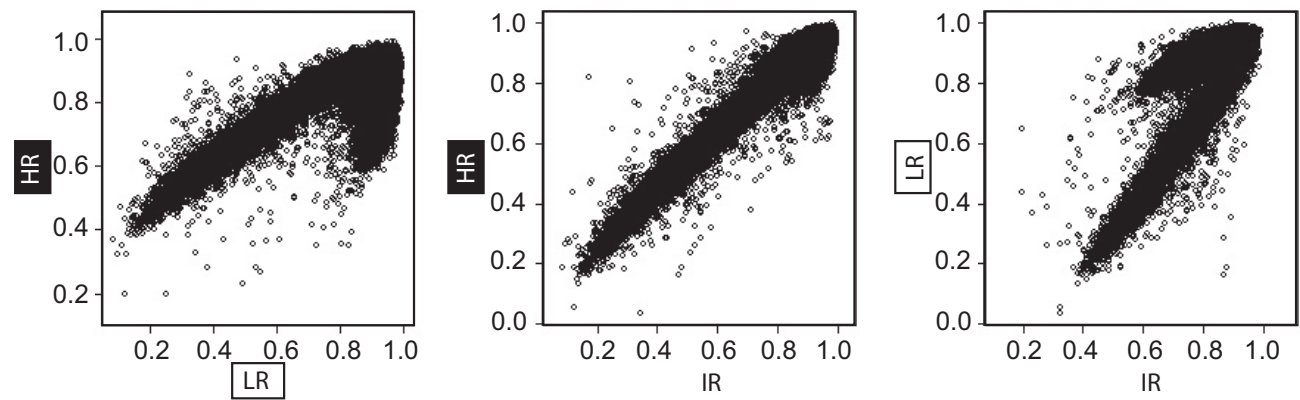

Figure 3. Contrasting DNA methylation patterns in the amygdala of $H R, L R$, and IR rats. Average DNA methylation profiles were created for $H R$, $L R$, and IR rats, which are known to exhibit an intermediate behavioral phenotype relative to the HR and LR extremes. Correlation analyses were then performed to evaluate the degree of similarity between IR and either HR or LR.

\begin{tabular}{|c|c|c|c|}
\hline & \multicolumn{2}{|l|}{ HR/IR DMR } & \\
\hline & Yes & No & \\
\hline \multicolumn{4}{|c|}{ LR/IR DMR } \\
\hline Yes & $n_{11}(9)$ & $n_{12}(172)$ & $\begin{array}{l}\text { No. of both HR/LR DMRs } \\
\text { and LR/IR DMRs }\end{array}$ \\
\hline \multirow[t]{3}{*}{ No } & $n_{21}(122)$ & $n_{22}(631)$ & $\begin{array}{l}\text { No. of HR/LR DMRs but } \\
\text { not LR/IR DMRs }\end{array}$ \\
\hline & No. of both HR/LR & No. of HR/LR & Total no. of of HR/LR \\
\hline & $\begin{array}{l}\text { DMRs and } \\
\text { HR/IR DMRs }\end{array}$ & $\begin{array}{l}\text { DMRs but not } \\
\text { HR/IR DMRs }\end{array}$ & DMRs: $n$ (934) \\
\hline
\end{tabular}

$\bar{a}$ Using the DMRs identified in HR rat versus $\mathrm{LR}$ rat methylome comparisons (threshold: $p<0.05$ ), the profile of $\mathrm{IR}$ rats, which are created by cross-breeding the HR/LR lines, was contrasted with HR and LR profiles (HR/IR or LR/IR comparisons). The paired outcomes are shown in a $2 \times 2$ contingency table. Because more LR/IR DMRs were observed than HR/IR DMRs (172 vs 122), the IR methylome profiles have a tendency to be more similar to HR ( $p=$ $0.004267 ;$ McNemar's $\chi^{2}$ test).

in part, to minimize stress of handing, injecting and/or performing surgery on pups, as these factors alone could impact neonatal brain development and ultimately offspring's emotional behavior.

Because the dietary manipulation was performed in mothers rather than the offspring themselves, an important control for this experiment was to determine whether dietary methyl donor depletion impacted maternal behavior. The diet manipulation began during late gestation (G17) and did not appear to impact litter size in either LR or HR dams. The average litter sizes for each group were as follows: LR-CON, $8.73 \pm 0.81$ pups; LR-DEP, $6.25 \pm 0.85$ pups; HR-CON, $13.60 \pm 0.42$ pups; and HR-DEP, $10.3 \pm 1.30$ pups). Maternal methyl donor depletion led to decreased body weight of offspring, which was consistent with a previous study using a similar diet manipulation (Konycheva et al., 2011). At P7, both male and female HR/LR pups in the pDEP group weighed less than control animals (male pDEP 6.171 \pm $0.352 \mathrm{~g}$; male pCON $14.310 \pm 0.710 \mathrm{~g}$; female pDEP $6.371 \pm$ $0.263 \mathrm{~g}$, female pCON $14.456 \pm 0.349 \mathrm{~g}$; main effect of maternal diet: $\left.F_{(1,28)}=231.9, p<0.0001\right)$.

In both phases of this experiment (the LR phase and then the HR phase), we monitored behavior of dams during the light phase and dark phase from P1 to P14. Table 2 provides a summary of data from these behavioral observations, displaying group means, SEM, and statistical results. In the LR portion of the experiment, we found that LR-DEP mothers displayed more arched back nursing, less licking/grooming, and a similar amount of passive nursing compared with LR-CON dams. In the HR phase of the study, HR-DEP dams showed less licking/grooming compared with the HR-CON group but displayed similar levels of arched-back nursing and passive nursing.
Adult male and female offspring of the CON and DEP dams (designated pCON and pDEP, respectively, to denote perinatal exposure to standard chow maternal control diet vs maternal methyl donor deprivation) were evaluated in a series of behavioral tests to assess emotional behavior. As noted above, the study was run in two phases to separately examine the effect of maternal methyl donor diet depletion on LR versus HR offspring.

Exposing LR offspring to perinatal methyl donor deprivation led to greater exploratory behavior in adulthood, with LR-pDEP offspring traversing greater distance in the OFT compared with control (Fig. $4 A, G$; for diet, $t_{(56)}=2.362, p=0.0217$; linear model with covariates sex, diet, weight). The LR-pDEP also showed a trend of less anxiety-like behavior in the OFT, spending more time in the center of the OF (Fig. $4 \mathrm{~B}, \mathrm{H}$; for diet, $t_{(56)}=$ $1.939, p=0.0576$; linear model with covariates sex, diet, weight). No differences found in time spent in open arms. In the Social Interaction Test, the LR-pDEP offspring displayed more social exploration than controls with female stimulus animals (Fig. $4 D, J$; for diet, $t_{(56)}=2.749, p=0.00803$; linear model with covariates sex, diet, weight). Additionally, the time spent with either the same sex versus the opposite sex were compared and female LR-pDEP spent more time with novel males than novel females (Fig. $4 B, H$; for experimental rat sex, $t_{(56)}=-2.641, p=$ 0.0006; for weight, $t_{(56)}=2.606, p=0.01173$; for diet, $t_{(56)}=$ 2.195, $p=0.03234$; linear model with covariates sex, diet, weight). In the FST, the LR-pDEP offspring displayed less depression-like behavior (immobility) compared with the LRpCON (Fig. $4 E, K$; for diet, $t_{(56)}=2.214, p=0.0309$; linear model with covariates sex, diet, weight). There was also a significant effect of weight on time spent immobile (Fig. $4 E, K$; for weight, $t_{(56)}=3.413, p=0.0012$; linear model with covariates sex, diet, weight). In the Sucrose Preference Test, both groups displayed similar preference (no effect of perinatal diet exposure and no diet $\times$ time interaction; Fig. $4 F, L)$.

Although the weights of the $\mathrm{pDEP} / \mathrm{pCON}$ were significantly different, there was only an effect on their behavioral profile in one measure, which was the time spent immobile in the FST. Immobility in the FST is known to often be affected by an animal's weight, with heavier animals tending to show more immobility than lighter ones. As for other factors that impact behavior, while sex often impacts behavioral measures, our analyses in the $\mathrm{pDEP} / \mathrm{pCON}$ animals did not reveal significant differences due to sex of the animal. Although the perinatal diet manipulation significantly improves LRs' anxiety/depression-like behavioral phenotype (relative to controls), it does not completely convert them to an HR-like phenotype; rather, perinatal methyl donordepleted LRs show behavior that more closely resembles IR rats, which exhibit an intermediate level of novelty-induced reactivity, 
Table 2. Impact of dietary methyl donor content on pregnancy outcomes and maternal behavior ${ }^{a}$

\begin{tabular}{|c|c|c|c|c|c|c|c|}
\hline & Phase & LR-pCON & LR-pDEP & Statistical effects & HR-pCON & HR-pDEP & Statistical effects \\
\hline \multirow[t]{2}{*}{ Arched-back nursing } & Light & $5.097 \pm 0.293$ & $7.177 \pm 0.346$ & ${ }^{b} p<0.0001, F_{(1,34)}=42.02$ & $4.357 \pm 0.348$ & $5.865 \pm 0.516$ & \multirow[t]{2}{*}{${ }^{c} p<0.0001, F_{(1,10)}=34.89$} \\
\hline & Dark & $2.28 \pm 0.205$ & $3.920 \pm 0.303$ & ${ }^{c} p=0.0113, F_{(1,34)}=112.1$ & $2.321 \pm 0.834$ & $1.539 \pm 0.610$ & \\
\hline \multirow[t]{2}{*}{ Passive nursing } & Light & $0.948 \pm 0.111$ & $1.025 \pm 0.265$ & NS & $2.881 \pm 0.438$ & $2.012 \pm 0.392$ & \multirow{2}{*}{${ }^{c} p<0.0001, F_{(1,10)}=62.42$} \\
\hline & Dark & $0.626 \pm 0.111$ & $1.161 \pm 0.160$ & & $0.272 \pm 0.165$ & $0.297 \pm 0.196$ & \\
\hline \multirow[t]{2}{*}{ Licking/grooming } & Light & $0.857 \pm 0.088$ & $0.387 \pm 0.048$ & ${ }^{b} p=0.0013, F_{(1,34)}=12.34$ & $1.190 \pm 0.214$ & $0.607 \pm 0.168$ & \multirow[t]{2}{*}{${ }^{b} p=0.0318, F_{(1,20)}=5.325$} \\
\hline & Dark & $0.955 \pm 0.102$ & $0.75 \pm 0.118$ & ${ }^{c} p=0.0222, F_{(1,34)}=5.745$ & $1.560 \pm 0.311$ & $0.842 \pm 0.383$ & \\
\hline \multirow[t]{2}{*}{ Self-directed behaviors (eat, self-groom, drink) } & Light & $0.266 \pm 0.0389$ & $0.237 \pm 0.036$ & ${ }^{b} p=0.0385, F_{(1,34)}=4.634$ & $1.083 \pm 0.184$ & $0.690 \pm 0.149$ & \multirow{4}{*}{$\begin{array}{l}{ }^{b} p=0.0061, F_{(1,10)}=12.00 \\
c_{p}=0.0005, F_{(1,10)}=25.33 \\
c_{p}=0.0251, F_{(1,10)}=2.227\end{array}$} \\
\hline & Dark & $1.097 \pm 0.083$ & $0.836 \pm 0.089$ & ${ }^{c} p<0.0001, F_{(1,34)}=112.3$ & $3.272 \pm 0.234$ & $1.956 \pm 0.496$ & \\
\hline \multirow[t]{2}{*}{ Resting away from nest } & Light & $2.688 \pm 0.231$ & $0.766 \pm 0.166$ & ${ }^{b} p<0.0001, F_{(1,34)}=60.34$ & $1.405 \pm 0.336$ & $0.548 \pm 0.342$ & \\
\hline & Dark & $2.319 \pm 0.179$ & $1.036 \pm 0.210$ & & $0.397 \pm 0.136$ & $0.344 \pm 0.208$ & \\
\hline
\end{tabular}

${ }^{a}$ Values are mean \pm SE for maternal behavior scores during the first 2 weeks postpartum for each experimental group. From gestational day 17 through weaning at postnatal day 21 , HR/LR dams were fed a DEP or CON. The experiment was run in two phases: an LR phase followed by an HR phase, applying the DEP/CON conditions to LR and HR dams, respectively. Data from two phases were analyzed separately. Groups were compared by two-way ANOVA, with diet and time of day (light or dark phase) as independent factors. NS, nonsignificant $p$ values $>0.05$ for statistical comparisons.

${ }^{b}$ Main effect of diet.

'Time (light or dark phase).

anxiety-like behavior, and other measures relative to the LR and HR extremes (Flagel et al., 2014).

When we repeated the perinatal methyl donor depletion experiment in HR offspring, we found that their adult behavioral phenotype was unaffected by perinatal diet condition, weight, or sex, although perinatal DEP treatment reduced the adult body weight of HR males and females (Fig. 4-1E, J, available at https:// doi.org/10.1523/JNEUROSCI.1157-15.2019.f4-1). For example, HR-pDEP and HR-pCON males showed similar levels of exploration in the OFT (Fig. 4-1A,F, available at https://doi.org/ 10.1523/JNEUROSCI.1157-15.2019.f4-1). HR-pDEP and HRpCON males showed similarly low levels of anxiety-like behavior in the OFT (Fig. 4-1B, G, available at https://doi.org/10.1523/ JNEUROSCI.1157-15.2019.f4-1) and EPM (Fig. 4-1C,H, available at https://doi.org/10.1523/JNEUROSCI.1157-15.2019.f4-1). In the FST, HR-pCON and HR-pDEP male group showed low levels of immobility (Fig. 4-1D, available at https://doi.org/ 10.1523/JNEUROSCI.1157-15.2019.f4-1). Perinatal methyl donor depletion had a similarly limited effect on behavior of HR females. Both pDEP and pCON female groups showed high levels of exploration in the OFT (Fig. 4-1 F, available at https://doi.org/ 10.1523/JNEUROSCI.1157-15.2019.f4-1). Both female groups showed low anxiety-like behavior in the OFT (Fig. 4-1G, available at https://doi.org/10.1523/JNEUROSCI.1157-15.2019.f4-1) and EPM (Fig. 4-1H, available at https://doi.org/10.1523/JNEUROSCI.115715.2019.f4-1). In FST, females of both groups displayed similar levels of immobility (Fig. 4-1I, available at https://doi.org/ 10.1523/JNEUROSCI.1157-15.2019.f4-1).

We chose to use the maternal methyl donor dietary deficiency approach since previous studies reported that it reduced DNA methylation markers in in offspring liver (Konycheva et al., 2011) and brain (Ishii et al., 2014). We were unable to confirm significant diet-induced global DNA methylation (5-methylcytosine) changes in amygdala samples collected from P21 pDEP and pCON offspring (pDEP $0.682 \pm 0.134 \%$ DNA methylation; pCON $0.832 \pm 0.345 \%$ DNA methylation). It is possible that dietary methyl donor depletion led to gene-specific methylation changes, but we were unable to detect such differences using the Epigentek assay.

Transiently suppressing DNMT3b expression in the developing amygdala produces greater novelty exploration and reduced anxiety in adult offspring

Because the perinatal methyl donor diet manipulation affects all body system development, we sought another approach to more specifically target DNA methylation in the early postnatal amygdala to test whether it could elicit lasting behavioral changes. Our earlier molecular studies pointed to increased global DNA methylation as well as increased levels of the DNMT, DNMT3b, in the amygdala of "high anxiety"-prone LR rats. Thus, this experiment used an siRNA approach to transiently suppress Dnmt3b expression in the developing amygdala to test whether it would lead to less anxiety- and/or depression-like behavior in adulthood. Notably, this experiment was conducted in IR rats because they are known to display a predictable intermediate behavioral phenotype.

To confirm efficacy of the siRNA-mediated Dnmt3b knockdown, a subset of Dnmt3b siRNA- and control siRNA-treated pups were killed $4 \mathrm{~d}$ after surgery. RNA samples were extracted from dissected amygdala to be used for TaqMan qPCR gene expression analysis as previously described (McCoy et al., 2016a). We found that the Dnmt3b siRNA treatment led to a $17 \%$ reduction of Dnmt3b levels compared with control siRNA-treated animals (control FC: $1.00 \pm 0.025$, KD FC: $-1.2 \pm 0.077$; one-tailed unpaired $t$ test: $t_{(7)}=1.9, p=0.0496$ ). In addition, we found that early-life Dnmt3b siRNA did not alter the gene expression of Dnmt1 and Dnmt3a gene expression at either the time of the knockdown or later in adulthood and did not alter Dnmt $3 b$ in adulthood when behavioral assessment was performed (threshold: $p<0.05)$.

For the animals that were kept for adult behavioral assessment, we examined weight of offspring $2 \mathrm{~d}$ following surgery as well as 6 additional times between weaning (P21) and adulthood (P75) when the behavioral test battery began. There were no group differences in weight at any of these time points.

In the OFT, adult male offspring that had been exposed to the early-life Dnmt3b knockdown (early-Dnmt3b KD) exhibited greater novelty exploration (Fig. $5 A ; t_{(26)}=2.810, p=0.0093$, unpaired $t$ test) and greater time spent in the center of the OF (Fig. $5 B ; t_{(20.87)}=3.158, p=0.0048$, Welch's corrected $t$ test) relative to control males. The developmental Dnmt3b knockdown also reduced males' anxiety-like behavior in the EPM, with early-Dnmt $3 b \mathrm{KD}$ males spending more time in the open arms compared with controls (Fig. $5 C ; t_{(17.02)}=3.061, p=0.0071$, Welch's corrected $t$ test). The early-life Dnmt $3 b$ manipulation did not affect males' behavior in the Social Interaction Test (Fig. 5D), FST (Fig. 5E), or Sucrose Preference Test (Fig. 5F).

The early-life Dnmt $3 b$ knockdown had a muted effect on adult female behavior. The early-Dnmt $3 b \mathrm{KD}$ and control females displayed similar levels of novelty exploration (Fig. 5G), anxiety-like 


\section{Males}

A
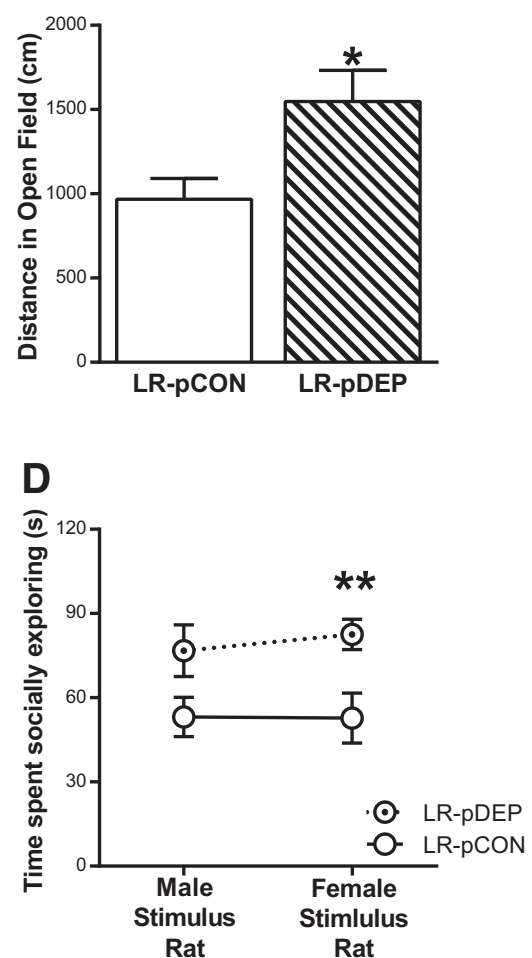

G
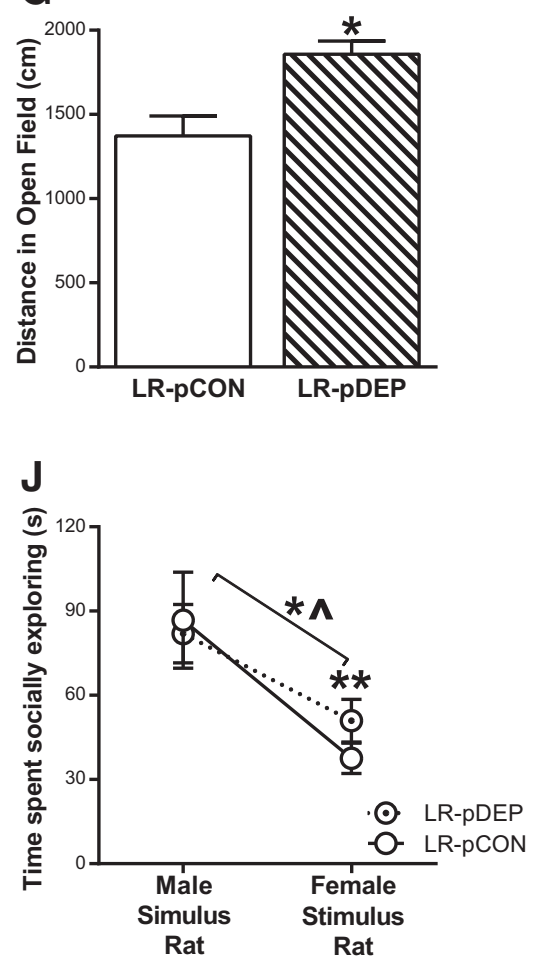

B

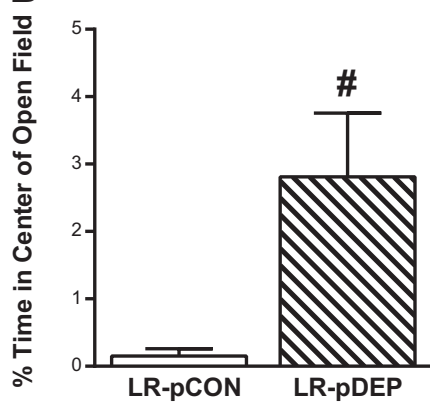

$\mathbf{E}$

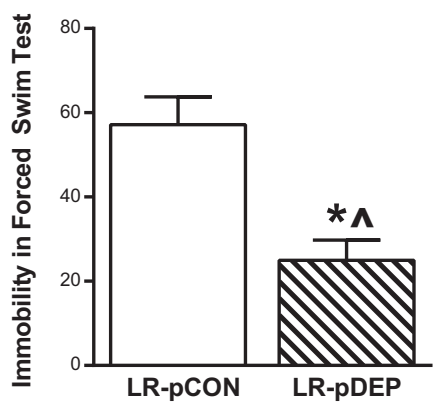

Females
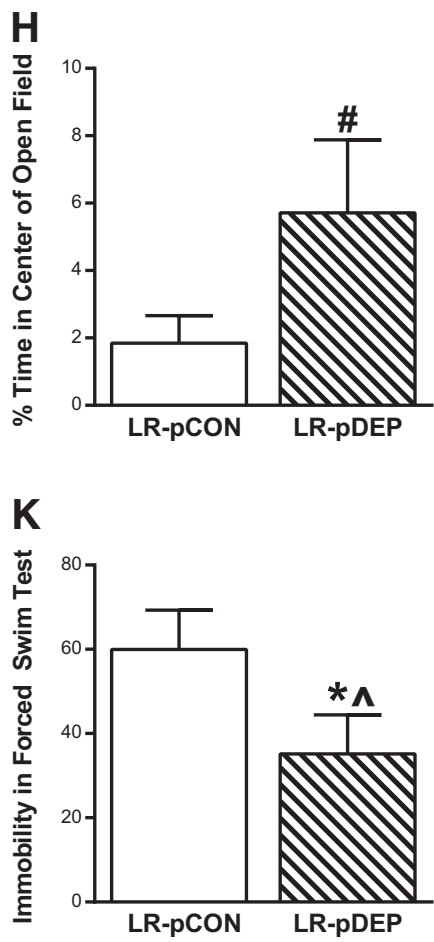
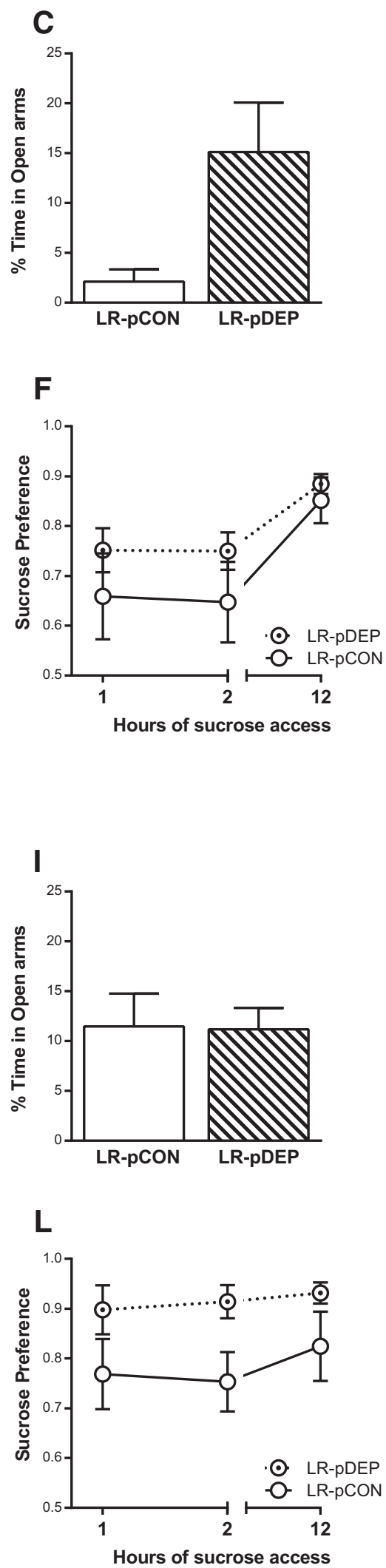

Figure 4. Perinatal methyl donor depletion improves $L R$ rats' adult anxiety- and depression-like behavior. In an attempt to reduce DNA methylation levels in developing $L R$ pups, $L R$ mothers were fed a diet severely depleted of methyl donors (or standard rat chow) from late pregnancy through weaning. Their adult offspring ( $p D E P$ and $p C O N ;$ males $n=15$, females $L R-p D E P n=14, L R-p C O N$ $n=16$ ) were later evaluated in several emotional behavior assays $(\boldsymbol{A}-\boldsymbol{L})$. $\boldsymbol{A}$, In male offspring, perinatal methyl donor deprivation led LRs to display greater exploratory behavior in the 0FT compared with controls. $B, C$, The LR-pDEP males also showed less anxiety-like behavior, with a trend to spend more time in the center of the open field and significantly more (Figure legend continues.) 
behavior in the OFT (Fig. 5H) and EPM (Fig. 5I), social interaction (Fig. $5 \mathrm{~J}$ ), and sucrose preference (Fig. $5 \mathrm{~L}$ ). The only significant effect of perinatal Dnmt3b knockdown on females was a slight but significant increase in FST immobility $\left(\right.$ Fig. $5 \mathrm{~K}$; $t_{(26)}=$ $2.225, p=0.0346)$.

\section{Discussion}

Myriad brain structure, function, and gene expression abnormalities occur in individuals with psychiatric disorders, such as depression and anxiety, but we are just beginning to recognize the role of epigenetic processes in these illnesses (Tsankova et al., 2007; Bernstein et al., 2010; Sabunciyan et al., 2012; Bagot et al., 2016). Growing evidence points to abnormal DNA methylation in the brains of psychiatric patients, but it is unknown whether such changes (1) represent inborn epigenetic aberrations that trigger psychopathology; (2) are secondary to factors, such as stress exposure; or (3) a combination of both. Model organisms, such as the HR/LR rats, offer a tool to dissect these factors and inform work in humans. Our recent transcriptome studies identified dramatic HR/LR gene expression differences in the early postnatal and adult amygdala (McCoy et al., 2016b, 2017). The present findings point to DNA methylation differences in the HR/LR amygdala that may contribute to their differences in developmental gene expression and behavior.

Our maternal diet manipulation and early-life Dnmt3b siRNA experiments aimed to lower DNA methylation levels in developing brain to test whether it would produce a low anxiety/ depression "HR-like" behavior in adulthood. Perinatal methyl donor depletion improved adult LR male and female offsprings' anxiety- and depression-like behavior. To target methylation in the amygdala specifically, we used an siRNA to suppress expression of DNMT3b in the developing amygdala of IR rats. Early-life Dnmt3b knockdown in amygdala led to reduced anxiety-like behavior in adult males but did not affect depression-like behavior and did not affect females' behavior, except increasing FST immobility. These data point to potential sex differences in DNA methylation in the developing brain (which we were unable to address as our present molecular studies were conducted in males only). There is little information on epigenetic differences in the male versus female brain (Menger et al., 2010; McCarthy and Nugent, 2015) and how such differences may contribute to sexually dimorphic risk for emotional dysfunction (Uddin et al., 2013). Thus, it will be important for future studies to determine whether both male and female individuals with high versus low propensity to an anxiety/depression-like phenotype display similar or unique differences in the limbic transcriptome/methylome and how those differences may impact behavior.

$\leftarrow$

(Figure legend continued.) time in the open arms of the EPM. D, Perinatal methyl donor depletion led male LRs to display more social exploration in the Social Interaction Test. $\boldsymbol{E}, \boldsymbol{F}$, During FST, male LR-pDEP show reduced immobility compared with LR-pCON, indicated less depression-like behavior, although the groups did not differ in the Sucrose Preference Test. FST was also significantly affected by the weight of the animal. G- $\mathbf{L}$, Perinatal methyl donor depletion elicited similar effects on LR females as it did in males, with female LR-pDEP offspring showing increased novelty-induced locomotion, more time spent in the center of the OF, less FST immobility and greater sucrose preference compared with LR-pCON females. Because the perinatal methyl donor deplete diet led to reduced body weight in exposed offspring, we used linear modeling for statistical analysis and considered diet, sex, and weight as factors. Through all behavioral measures, sex was not found to have a significant effect and weight only have a significant effect on FST. Last, the perinatal methyl donor depletion study was repeated in HR offspring, which had limited effect on their behavioral phenotype (Figure 4-1, available at https://doi.org/10.1523/JNEUROSCI.1157-15.2019.f4-1). ${ }^{\#} p=0.0576$; ${ }^{*} p<0.05 ;{ }^{* *} p<$ 0.01 ; compared with control. $\wedge p<0.05$, considering effect of weight.

\section{High anxiety/depression-behavior prone LR rats exhibit} elevated DNA methylation in the early postnatal amygdala

Numerous studies in the bred HR/LR rats demonstrate their disparate behavioral phenotypes, with LR rats typically exhibiting high levels of anxiety- and depression-like behavior compared with HRs (for review, see Flagel et al., 2014). These traits emerge during early life and are likely linked to disparate development of the hippocampus (Clinton et al., 2011; Turner et al., 2011) and amygdala (Simmons et al., 2012; McCoy et al., 2016b). Our current data suggest that altered DNA methylation in the developing amygdala may be a molecular mechanism that contributes to HR/LR transcriptome and behavioral differences.

We found increased DNA methylation levels, altered DNMT expression, and gene-specific methylation changes in the LR versus $\mathrm{HR}$ amygdala at $\mathrm{P} 7$, with the alterations dissipating by the second and third weeks of life. The P7 methylation differences coincide with a key neurodevelopmental transition period in the amygdala. Although neurogenesis in amygdala peaks during mid-gestation (Berger et al., 2002), at P7, neurons migrate to amygdala to begin subnuclei organization (Berdel et al., 1997). The P7-P28 timeframe is characterized by high levels of synaptogenesis and dendritic expansion in the amygdala (Escobar and Salas, 1993; Morys et al., 1998; Ryan et al., 2016), which reflects increased connectivity within amygdalar subregions and increased inputs from areas, such as the prefrontal cortex (Bouwmeester et al., 2002). Such neurodevelopmental events are accompanied by massive transcriptome changes (Mody et al., 2001; Stead et al., 2006a; Liscovitch and Chechik, 2013; Thompson et al., 2014), and our prior microarray study highlighted numerous HR/LR gene expression differences in the developing amygdala, including genes involved in neurodevelopment, synaptogenesis, and metabolism (McCoy et al., 2016b). It seems conceivable that DNA methylation differences in the P7 HR/LR amygdala could provide a "molecular switch" that impacts gene expression and related neurodevelopmental processes, thereby altering trajectory of amygdalar development in HR/LR rats.

Our next-generation sequencing methylome analysis revealed a preponderance of genomic sites that were hypermethylated in LR versus HR samples, which is consistent with our finding of increased global methylation in the P7 LR versus HR amygdala. Over half of the differentially methylated sites occurred within 10 $\mathrm{kb}$ of genes, which suggests that they could play a role in transcriptional activity. The top molecular pathways showing evidence of differential DNA methylation involved genes related to: (1) glutamate neurotransmission, including both NMDAR and AMPAR signaling; and (2) Rap1 and PI3K-Akt signaling. Both pathways could influence cell migration, which could align with the notion of disparate developmental trajectories in the HR/LR amygdala. Future studies should explore structural and functional differences that may emerge in the developing HR versus LR amygdala and determine how these may contribute to their disparate behavioral phenotypes.

\section{Manipulating DNA methylation in developing LR rats leads to improved adult anxiety- and depression-like behavior} Dietary folate, choline, and methionine act as methyl donors for one-carbon transfer reactions, such as DNA methylation. DNMTs transfer methyl groups from S-adenosylmethionine to cytosine (Jones and Takai, 2001), so diets lacking methyl donors impede S-adenosylmethionine synthesis and reduce DNA methylation (Ghoshal et al., 2006; Pogribny et al., 2008; Chen et al., 2010). Because we observed increased DNA methylation in the developing LR versus HR brain, we hypothesized that decreasing 


\section{Males}

A
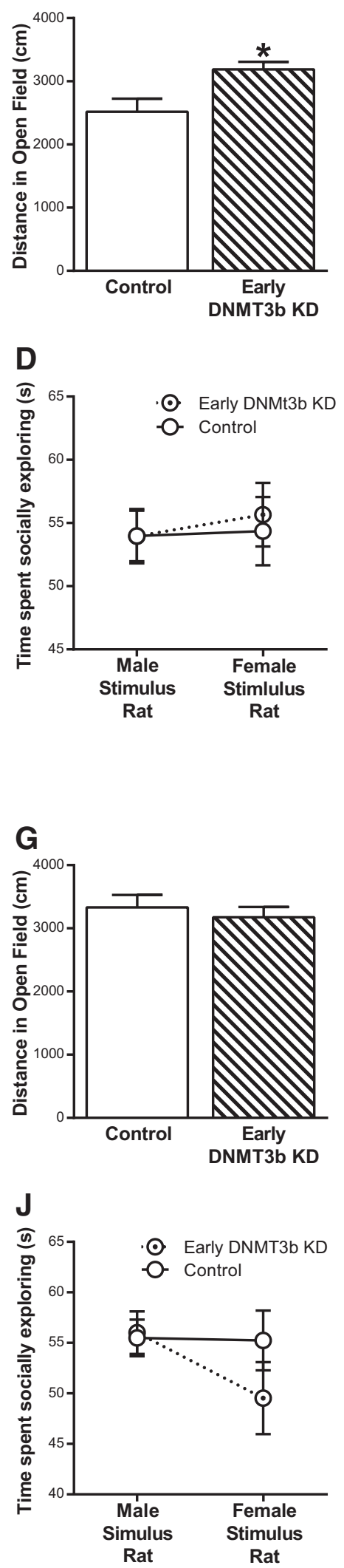
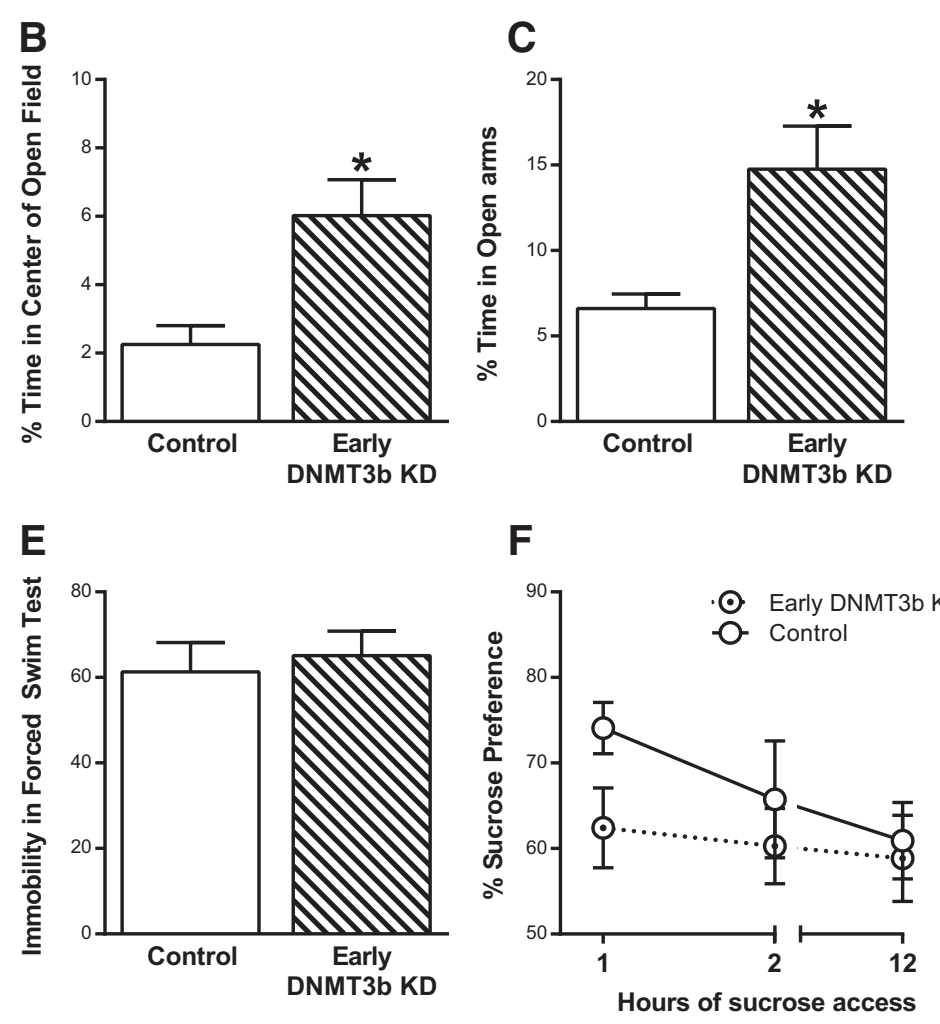

\section{Females}
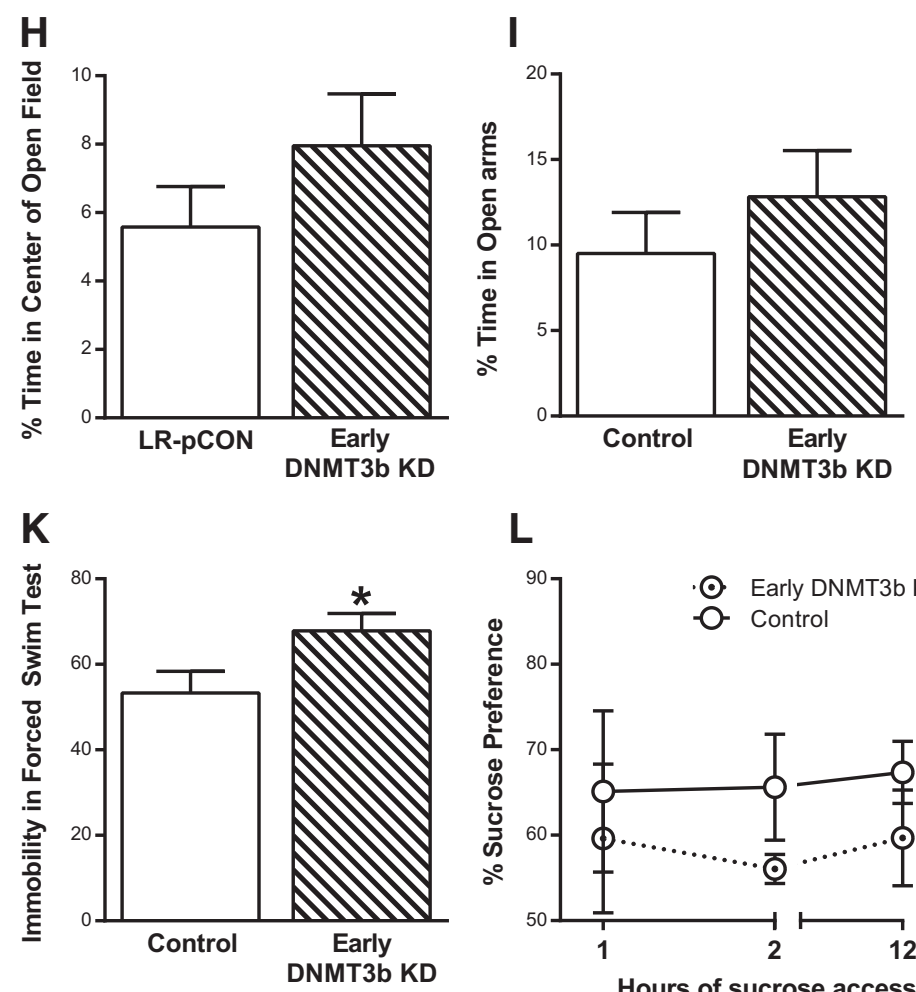

L

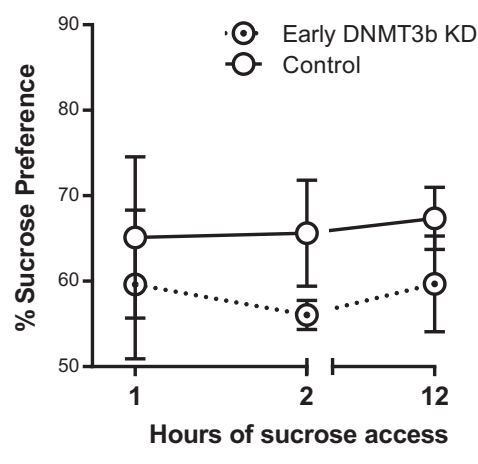

Figure 5. Transiently suppressing Dnmt3b expression in the developing amygdala leads to reduced anxiety-like behavior in adulthood. An siRNA was injected into the early postnatal amygdala to transiently suppress expression of the DNMT, Dnmt3b, to test whether this would lead to less anxiety- and/or depression-like behavior in adulthood (Dnmt $3 \mathrm{~b}$ siRNA: $n=15$ males; $n=14$ females; control siRNA $n=14$ males; $n=15$ females; $\boldsymbol{A}-\boldsymbol{L}$ ). $\boldsymbol{A}$, In males, early-life DNMT3b knockdown (KD) leads to greater novelty-induced exploration in adult (Figure legend continues.) 
DNA methylation in LR neonates (by altering mothers' dietary methyl donor content) could improve offsprings' adult anxietyand/or depression-like behavior. We found that, indeed, depleting maternal dietary methyl donor content shifted adult LR male/ female offspring behavior, leading to greater novelty exploration, decreased anxiety- and depression-like behavior. Maternal methyl donor depletion had little effect on HR offspring behavior.

Previous studies using maternal dietary methyl donor depletion reported mixed results on offspring behavior. Two studies found that it improved offspring learning and memory function (Plyusnina et al., 2007; Konycheva et al., 2011). One study in mice reported reduced locomotor activity in offspring exposed to perinatal methyl donor depletion (Middaugh et al., 1976). Another group reported increased anxiety in perinatal methyl depleted offspring, although the effect was only apparent in females (Konycheva et al., 2011). Together with our current results, it is tempting to speculate that manipulations of methyl donor dietary content may evoke distinct results depending upon baseline individual differences in DNA methylation. That said, it is also important to consider multiple ways that maternal diet manipulation could affect offspring. Our data show a significant weight difference in perinatal methyl donor depleted offspring and found that maternal methyl donor depletion affected maternal behavior, which itself could impact offspring's later emotional behavior. Because of these potential confounds, we chose to add the siRNA experiment as another method to manipulate DNA methylation in developing animals.

\section{Technical considerations and limitations}

An important consideration for the observed DNA methylation differences in the early postnatal HR/LR amygdala is whether they (1) represent basic neurobiological differences between the HR/LR lines, or (2) are driven, in part, by known HR/LR maternal behavior differences (Clinton et al., 2007, 2010). Previous cross-fostering studies by our laboratory group showed that cross-fostering LR pups to HR mothers shifted amygdalar gene expression and reduced adult anxiety-like behavior (Cohen et al., 2015). Preliminary studies indicate that those cross-fostering effects are not mediated by changes in DNA methylation, although future studies will be required to examine this possibility more closely. Last, a limit of the maternal methyl donor dietary experiment is that we were unable to confirm a diet-induced reduction of global methylation in the P7 amygdala, which conflicts with prior reports of this diet reducing DNA methylation in offspring liver (Konycheva et al., 2011) and reducing Dnmt3a and Dnmt3b mRNA in offspring brain (Ishii et al., 2014). It is possible that the diet produced gene-specific methylation changes that were not detectable using the assay that we chose. These technical difficulties motivated the follow-up manipulation experiment using Dnmt3b siRNA, which permitted transient Dnmt3b knockdown in the developing amygdala.

In conclusion, the roots of mental illnesses, such as depression and anxiety, are thought to involve aberrant brain development

\footnotetext{
$\leftarrow$

(Figure legend continued.) animals compared with controls. B, C, The siRNA Dnmt3b KD males also showed less anxiety-like behavior, spending more time in the center of the open field and more time in the open arms of the EPM. D-F, The early-life DNMT3b KD did not affect male rats' behavior in the Social Interaction Test, FST, or Sucrose Preference Test. G-L, Female animals were less affected by the transient early-life DNMT3b KD with the early DNMT3b KD females and control females displaying similar behavior across all tests, except increased immobility in FST. ${ }^{*} p<0.05$ compared with control.
}

(Leonardo and Hen, 2008; Monk, 2008; Pine and Fox, 2015). Future therapeutic strategies may be highly effective if applied during early life while the brain is highly plastic and before a mental illness takes hold. There is promising evidence for early behavioral interventions benefiting children who display high levels of behavioral inhibition and are at risk for internalizing disorders (Rapee, 2013; Frenkel et al., 2015). Studies using animal models of emotional dysfunction offer the possibility to understand the mechanisms of these effects and the chance to uncover new therapeutic opportunities. Our results suggest that individual differences in the developing DNA methylome trigger altered limbic system development and a high depression/anxiety-like phenotype, offering a glimpse at an epigenetic mechanism that may contribute to risk for developing emotional disorders.

\section{References}

Bagot RC, Cates HM, Purushothaman I, Lorsch ZS, Walker DM, Wang J, Huang X, Schlüter OM, Maze I, Peña CJ, Heller EA, Issler O, Wang M, Song WM, Stein JL, Liu X, Doyle MA, Scobie KN, Sun HS, Neve RL, et al. (2016) Circuit-wide transcriptional profiling reveals brain regionspecific gene networks regulating depression susceptibility. Neuron 90:969-983.

Bedrosian TA, Quayle C, Novaresi N, Gage FH (2018) Early life experience drives structural variation of neural genomes in mice. Science 359:13951399.

Berdel B, Morys J, Maciejewska B (1997) Neuronal changes in the basolateral complex during development of the amygdala of the rat. Int J Dev Neurosci 15:755-765.

Berger MA, Barros VG, Sarchi MI, Tarazi FI, Antonelli MC (2002) Longterm effects of prenatal stress on dopamine and glutamate receptors in adult rat brain. Neurochem Res 27:1525-1533.

Bernstein BE, Stamatoyannopoulos JA, Costello JF, Ren B, Milosavljevic A, Meissner A, Kellis M, Marra MA, Beaudet AL, Ecker JR, Farnham PJ, Hirst M, Lander ES, Mikkelsen TS, Thomson JA (2010) The NIH roadmap epigenomics mapping consortium. Nat Biotechnol 28:1045-1048.

Biederman J, Hirshfeld-Becker DR, Rosenbaum JF, Hérot C, Friedman D, Snidman N, Kagan J, Faraone SV (2001) Further evidence of association between behavioral inhibition and social anxiety in children. Am J Psychiatry 158:1673-1679.

Blankenberg D, Von Kuster G, Coraor N, Ananda G, Lazarus R, Mangan M, Nekrutenko A, Taylor J (2010) Galaxy: a web-based genome analysis tool for experimentalists. Curr Protoc Mol Biol Chapter 19:Unit 19.10.121.

Bock C, Walter J, Paulsen M, Lengauer T (2008) Inter-individual variation of DNA methylation and its implications for large-scale epigenome mapping. Nucleic Acids Res 36:e55.

Bouwmeester H, Smits K, Van Ree JM (2002) Neonatal development of projections to the basolateral amygdala from prefrontal and thalamic structures in rat. J Comp Neurol 450:241-255.

Brinkman AB, Simmer F, Ma K, Kaan A, Zhu J, Stunnenberg HG (2010) Whole-genome DNA methylation profiling using MethylCap-seq. Methods 52:232-236.

Chen NC, Yang F, Capecci LM, Gu Z, Schafer AI, Durante W, Yang XF, Wang $\mathrm{H}$ (2010) Regulation of homocysteine metabolism and methylation in human and mouse tissues. FASEB J 24:2804-2817.

Clinton SM, Vázquez DM, Kabbaj M, Kabbaj MH, Watson SJ, Akil H (2007) Individual differences in novelty-seeking and emotional reactivity correlate with variation in maternal behavior. Horm Behav 51:655-664.

Clinton SM, Miller S, Watson SJ, Akil H (2008) Prenatal stress does not alter innate novelty-seeking behavioral traits, but differentially affects individual differences in neuroendocrine stress responsivity. Psychoneuroendocrinology 33:162-177.

Clinton SM, Bedrosian TA, Abraham AD, Watson SJ, Akil H (2010) Neural and environmental factors impacting maternal behavior differences in high- versus low-novelty-seeking rats. Horm Behav 57:463-473.

Clinton SM, Stead JD, Miller S, Watson SJ, Akil H (2011) Developmental underpinnings of differences in rodent novelty-seeking and emotional reactivity. Eur J Neurosci 34:994-1005.

Cohen JL, Glover ME, Pugh PC, Fant AD, Simmons RK, Akil H, Kerman IA, Clinton SM (2015) Maternal style selectively shapes amygdalar develop- 
ment and social behavior in rats genetically prone to high anxiety. Dev Neurosci 37:203-214.

Cryan JF, Valentino RJ, Lucki I (2005) Assessing substrates underlying the behavioral effects of antidepressants using the modified rat forced swimming test. Neurosci Biobehav Rev 29:547-569.

Cummings JA, Clinton SM, Perry AN, Akil H, Becker JB (2013) Male rats that differ in novelty exploration demonstrate distinct patterns of sexual behavior. Behav Neurosci 127:47-58.

Dempster EL, Pidsley R, Schalkwyk LC, Owens S, Georgiades A, Kane F, Kalidindi S, Picchioni M, Kravariti E, Toulopoulou T, Murray RM, Mill J (2011) Disease-associated epigenetic changes in monozygotic twins discordant for schizophrenia and bipolar disorder. Hum Mol Genet 20:4786-4796.

Escobar C, Salas M (1993) Neonatal undernutrition and amygdaloid nuclear complex development: an experimental study in the rat. Exp Neurol 122:311-318.

Flagel SB, Waselus M, Clinton SM, Watson SJ, Akil H (2014) Antecedents and consequences of drug abuse in rats selectively bred for high and low response to novelty. Neuropharmacology 76:425-436.

Frenkel TI, Fox NA, Pine DS, Walker OL, Degnan KA, Chronis-Tuscano A (2015) Early childhood behavioral inhibition, adult psychopathology and the buffering effects of adolescent social networks: a twenty-year prospective study. J Child Psychol Psychiatry 56:1065-1073.

Ghoshal K, Li X, Datta J, Bai S, Pogribny I, Pogribny M, Huang Y, Young D, Jacob ST (2006) A folate- and methyl-deficient diet alters the expression of DNA methyltransferases and methyl CpG binding proteins involved in epigenetic gene silencing in livers of F344 rats. J Nutr 136:1522-1527.

Giardine B, Riemer C, Hardison RC, Burhans R, Elnitski L, Shah P, Zhang Y, Blankenberg D, Albert I, Taylor J, Miller W, Kent WJ, Nekrutenko A (2005) Galaxy: a platform for interactive large-scale genome analysis. Genome Res 15:1451-1455.

Goecks J, Nekrutenko A, Taylor J (2010) Galaxy: a comprehensive approach for supporting accessible, reproducible, and transparent computational research in the life sciences. Genome Biol 11:R86.

Ishii D, Matsuzawa D, Matsuda S, Tomizawa H, Sutoh C, Shimizu E (2014) Methyl donor-deficient diet during development can affect fear and anxiety in adulthood in C57BL/6J mice. PLoS One 9:e105750.

Jones PA, Takai D (2001) The role of DNA methylation in mammalian epigenetics. Science 293:1068-1070.

Kagan J, Reznick JS, Snidman N (1987) The physiology and psychology of behavioral inhibition in children. Child Dev 58:1459-1473.

Konycheva G, Dziadek MA, Ferguson LR, Krägeloh CU, Coolen MW, Davison M, Breier BH (2011) Dietary methyl donor deficiency during pregnancy in rats shapes learning and anxiety in offspring. Nutr Res 31:790804.

Kuleshov MV, Jones MR, Rouillard AD, Fernandez NF, Duan Q, Wang Z, Koplev S, Jenkins SL, Jagodnik KM, Lachmann A, McDermott MG, Monteiro CD, Gundersen GW, Ma'ayan A (2016) Enrichr: a comprehensive gene set enrichment analysis web server 2016 update. Nucleic Acids Res 44:W90-W97.

LaSalle JM (2011) A genomic point-of-view on environmental factors influencing the human brain methylome. Epigenetics 6:862-869.

Leonardo ED, Hen R (2008) Anxiety as a developmental disorder. Neuropsychopharmacology 33:134-140.

Lienhard M, Grimm C, Morkel M, Herwig R, Chavez L (2014) MEDIPS: genome-wide differential coverage analysis of sequencing data derived from DNA enrichment experiments. Bioinformatics 30:284-286.

Liscovitch N, Chechik G (2013) Specialization of gene expression during mouse brain development. PLoS Comput Biol 9:e1003185.

McCarthy MM, Nugent BM (2015) At the frontier of epigenetics of brain sex differences. Front Behav Neurosci 9:221.

McCoy CR, Rana S, Stringfellow SA, Day JJ, Wyss JM, Clinton SM, Kerman IA (2016a) Neonatal maternal separation stress elicits lasting DNA methylation changes in the hippocampus of stress-reactive Wistar Kyoto rats. Eur J Neurosci 44:2829-2845.

McCoy CR, Golf SR, Melendez-Ferro M, Perez-Costas E, Glover ME, Jackson NL, Stringfellow SA, Pugh PC, Fant AD, Clinton SM (2016b) Altered metabolic activity in the developing brain of rats predisposed to high versus low depression-like behavior. Neuroscience 324:469-484.

McCoy CR, Jackson NL, Day J, Clinton SM (2017) Genetic predisposition to high anxiety- and depression-like behavior coincides with diminished
DNA methylation in the adult rat amygdala. Behav Brain Res 320:165-178.

McGowan PO, Sasaki A, D’Alessio AC, Dymov S, Labonté B, Szyf M, Turecki G, Meaney MJ (2009) Epigenetic regulation of the glucocorticoid receptor in human brain associates with childhood abuse. Nat Neurosci 12:342-348.

Menger Y, Bettscheider M, Murgatroyd C, Spengler D (2010) Sex differences in brain epigenetics. Epigenomics 2:807-821.

Middaugh LD, Grover TA, Blackwell LA, Zemp JW (1976) Neurochemical and behavioral effects of diet related perinatal folic acid restriction. Pharmacol Biochem Behav 5:129-134.

Mill J, Tang T, Kaminsky Z, Khare T, Yazdanpanah S, Bouchard L, Jia P, Assadzadeh A, Flanagan J, Schumacher A, Wang SC, Petronis A (2008) Epigenomic profiling reveals DNA-methylation changes associated with major psychosis. Am J Hum Genet 82:696-711.

Mitchnick KA, Creighton S, O'Hara M, Kalisch BE, Winters BD (2015) Differential contributions of de novo and maintenance DNA methyltransferases to object memory processing in the rat hippocampus and perirhinal cortex: a double dissociation. Eur J Neurosci 41:773-786.

Mody M, Cao Y, Cui Z, Tay KY, Shyong A, Shimizu E, Pham K, Schultz P, Welsh D, Tsien JZ (2001) Genome-wide gene expression profiles of the developing mouse hippocampus. Proc Natl Acad Sci U S A 98:88628867.

Monk CS (2008) The development of emotion-related neural circuitry in health and psychopathology. Dev Psychopathol 20:1231-1250.

Moore H, Jentsch JD, Ghajarnia M, Geyer MA, Grace AA (2006) A neurobehavioral systems analysis of adult rats exposed to methylazoxymethanol acetate on E17: implications for the neuropathology of schizophrenia. Biol Psychiatry 60:253-264.

Moore LD, Le T, Fan G (2013) DNA methylation and its basic function. Neuropsychopharmacology 38:23-38.

Morys J, Berdel B, Kowianski P, Dziewiatkowski J (1998) The pattern of synaptophysin changes during the maturation of the amygdaloid body and hippocampal hilus in the rat. Folia Neuropathol 36:15-23.

Murgatroyd C, Patchev AV, Wu Y, Micale V, Bockmühl Y, Fischer D, Holsboer F, Wotjak CT, Almeida OF, Spengler D (2009) Dynamic DNA methylation programs persistent adverse effects of early-life stress. Nat Neurosci 12:1559-1566.

Nakajima H, Kubo T, Semi Y, Itakura M, Kuwamura M, Izawa T, Azuma YT, Takeuchi T (2012) A rapid, targeted, neuron-selective, in vivo knockdown following a single intracerebroventricular injection of a novel chemically modified siRNA in the adult rat brain. J Biotechnol 157:326333.

Nam H, Clinton SM, Jackson NL, Kerman IA (2014) Learned helplessness and social avoidance in the Wistar-Kyoto rat. Front Behav Neurosci 8:109.

Naumova OY, Lee M, Koposov R, Szyf M, Dozier M, Grigorenko EL (2012) Differential patterns of whole-genome DNA methylation in institutionalized children and children raised by their biological parents. Dev Psychopathol 24:143-155.

Pine DS, Fox NA (2015) Childhood antecedents and risk for adult mental disorders. Annu Rev Psychol 66:459-485.

Plyusnina IZ, Os'kina IN, Shchepina OA, Prasolova LA, Trut LN (2007) A maternal methyl-containing diet alters learning ability in the Morris swimming test in adult rats. Neurosci Behav Physiol 37:425-428.

Pogribny IP, Karpf AR, James SR, Melnyk S, Han T, Tryndyak VP (2008) Epigenetic alterations in the brains of Fisher 344 rats induced by longterm administration of folate/methyl-deficient diet. Brain Res 1237:2534.

Porsolt RD, Le Pichon M, Jalfre M (1977) Depression: a new animal model sensitive to antidepressant treatments. Nature 266:730-732.

Rakyan VK, Beck S (2006) Epigenetic variation and inheritance in mammals. Curr Opin Genet Dev 16:573-577.

Rapee RM (2013) The preventative effects of a brief, early intervention for preschool-aged children at risk for internalising: follow-up into middle adolescence. J Child Psychol Psychiatry 54:780-788.

Richards EJ (2008) Population epigenetics. Curr Opin Genet Dev 18:221226.

Rosenbaum JF, Biederman J, Hirshfeld-Becker DR, Kagan J, Snidman N, Friedman D, Nineberg A, Gallery DJ, Faraone SV (2000) A controlled study of behavioral inhibition in children of parents with panic disorder and depression. Am J Psychiatry 157:2002-2010. 
Roth TL, Lubin FD, Funk AJ, Sweatt JD (2009) Lasting epigenetic influence of early-life adversity on the BDNF gene. Biol Psychiatry 65:760-769.

Ryan SJ, Ehrlich DE, Rainnie DG (2016) Morphology and dendritic maturation of developing principal neurons in the rat basolateral amygdala. Brain Struct Funct 221:839-854.

Sabunciyan S, Aryee MJ, Irizarry RA, Rongione M, Webster MJ, Kaufman WE, Murakami P, Lessard A, Yolken RH, Feinberg AP, Potash JB (2012) Genome-wide DNA methylation scan in major depressive disorder. PLoS One 7:e34451.

Simmons RK, Howard JL, Simpson DN, Akil H, Clinton SM (2012) DNA methylation in the developing hippocampus and amygdala of anxietyprone versus risk-taking rats. Dev Neurosci 34:58-67.

Simmons RK, Stringfellow SA, Glover ME, Wagle AA, Clinton SM (2013) DNA methylation markers in the postnatal developing rat brain. Brain Res 1533:26-36.

Stead JD, Neal C, Meng F, Wang Y, Evans S, Vazquez DM, Watson SJ, Akil H (2006a) Transcriptional profiling of the developing rat brain reveals that the most dramatic regional differentiation in gene expression occurs postpartum. J Neurosci 26:345-353.

Stead JD, Clinton S, Neal C, Schneider J, Jama A, Miller S, Vazquez DM, Watson SJ, Akil H (2006b) Selective breeding for divergence in noveltyseeking traits: heritability and enrichment in spontaneous anxiety-related behaviors. Behav Genet 36:697-712.

Stedenfeld KA, Clinton SM, Kerman IA, Akil H, Watson SJ, Sved AF (2011)
Novelty-seeking behavior predicts vulnerability in a rodent model of depression. Physiol Behav 103:210-216.

Sun H, Kennedy PJ, Nestler EJ (2013) Epigenetics of the depressed brain: role of histone acetylation and methylation. Neuropsychopharmacology 38:124-137.

Thompson CL, Ng L, Menon V, Martinez S, Lee CK, Glattfelder K, Sunkin SM, Henry A, Lau C, Dang C, Garcia-Lopez R, Martinez-Ferre A, Pombero A, Rubenstein JL, Wakeman WB, Hohmann J, Dee N, Sodt AJ, Young R, Smith K, et al. (2014) A high-resolution spatiotemporal atlas of gene expression of the developing mouse brain. Neuron 83:309-323.

Tsankova N, Renthal W, Kumar A, Nestler EJ (2007) Epigenetic regulation in psychiatric disorders. Nat Rev Neurosci 8:355-367.

Turner CA, Clinton SM, Thompson RC, Watson SJ Jr, Akil H (2011) Fibroblast growth factor-2 (FGF2) augmentation early in life alters hippocampal development and rescues the anxiety phenotype in vulnerable animals. Proc Natl Acad Sci U S A 108:8021-8025.

Uddin M, Sipahi L, Li J, Koenen KC (2013) Sex differences in DNA methylation may contribute to risk of PTSD and depression: a review of existing evidence. Depress Anxiety 30:1151-1160.

Zhang D, Cheng L, Badner JA, Chen C, Chen Q, Luo W, Craig DW, Redman M, Gershon ES, Liu C (2010) Genetic control of individual differences in gene-specific methylation in human brain. Am J Hum Genet 86:411419. 